\title{
Electromagnetic waves around the proton cyclotron frequency in the sheath regions of interplanetary magnetic clouds: STEREO observations
}

\author{
Q. H. Li, L. Yang, D. J. Wu and T. Y. Wang
}

\section{Published version information}

Citation: QH Li et al. "Electromagnetic waves around the proton cyclotron frequency in the sheath regions of interplanetary magnetic clouds: STEREO observations." Astrophysical Journal, vol. 874, no. 1 (2019): 55.

DOI: $\underline{10.3847 / 1538-4357 / a b 06 f 7}$

This version is made available in accordance with publisher policies. Please cite only the published version using the reference above. This is the citation assigned by the publisher at the time of issuing the APV. Please check the publisher's website for any updates. 


\title{
Electromagnetic Waves around the Proton Cyclotron Frequency in the Sheath Regions of Interplanetary Magnetic Clouds: STEREO Observations
}

\author{
Q. H. Li ${ }^{1,2}$, L. Yang ${ }^{1,3}$, D. J. Wu ${ }^{1}$ (i), and T. Y. Wang ${ }^{4}$ \\ ${ }^{1}$ Key Laboratory of Planetary Sciences, Purple Mountain Observatory, Chinese Academy of Sciences, Nanjing 210034, People’s Republic of China; ylei@pmo.ac.cn \\ ${ }^{2}$ University of Science and Technology of China, Hefei 230026, People's Republic of China \\ ${ }^{3}$ State Key Laboratory of Space Weather, National Space Science Center, CAS, Beijing 100190, People's Republic of China \\ ${ }^{4}$ RAL Space, Rutherford Appleton Laboratory, Harwell Oxford, Didcot OX11 0QX, UK \\ Received 2018 September 24; revised 2019 January 24; accepted 2019 February 12; published 2019 March 21
}

\begin{abstract}
The compressed and turbulent sheath regions of interplanetary magnetic clouds (IMCs) provide a natural laboratory to study electromagnetic waves (EMWs) around the proton cyclotron frequency $f_{\mathrm{cp}}$. Based on the Morlet wavelet spectral analysis, the repeated filtering analysis and the minimum variance analysis of high-resolution magnetic field data from the STEREO spacecraft, 81 EMW events are identified in the sheath regions of six IMCs. These EMWs are all transverse, almost circularly polarized, and quasi-parallel propagating along the background magnetic field $\boldsymbol{B}_{0}$. They can be left-handed (LH) or right-handed (RH) polarized in the spacecraft frame, where the occurrence rate of the LH-polarized EMWs is higher than that of RH-polarized ones, consistent with previous observations in the solar wind. Also, a comparative analysis of polarization sense of these EMWs has been made in the spacecraft and plasma frames. Our results show that more than half of EMW events suffer a polarization reversal from the spacecraft to plasma frames, which are deduced to propagate inward relative to the solar wind flow. Others are outward-propagating waves. In the plasma frame, the outward-propagating LH-EMWs and inward-propagating RH-EMWs have relatively higher occurrence rates than the inward-propagating LH-EMWs and outward-propagating RH-EMWs, respectively. Furthermore, in the plasma frame all the frequencies of LHEMWs are below $f_{\mathrm{cp}}$, but the RH-EMW frequencies can exceed $f_{\mathrm{cp}}$. These results are helpful in understanding the physical properties of EMWs and their roles in the sheath regions of IMCs.
\end{abstract}

Key words: instabilities - magnetic fields - Sun: coronal mass ejections (CMEs) - solar wind - waves

\section{Introduction}

Electromagnetic waves (EMWs) around the proton cyclotron frequency play an important role in modifying the background plasma dynamics and energizing particles in collisionless plasmas (Hollweg 1975; Marsch 2006). By studying these waves, one can acquire the information of plasma properties or the related free energy sources. In the case of the parallel propagation $\left(\boldsymbol{k} \times \boldsymbol{B}_{0}=0\right.$, where $\boldsymbol{k}$ and $\boldsymbol{B}_{0}$ are the wave vector and the background magnetic field, respectively), these EMWs are circularly polarized transverse waves (Stix 1962; Gary 1993; Omidi et al. 2011). Generally their polarization handedness can be left-handed (LH) or right-handed (RH) in the plasma frame (Gary 1993), and different mechanisms are responsible for the generation and excitation of LH- and RHEMWs. In space plasmas, the LH instability can be driven by a variety of free energy sources, such as proton temperature anisotropy with the perpendicular temperature $T_{\perp p}$ larger than the parallel temperature $T_{\| p}$ (where the subscripts $\perp$ and $\|$ denote directions with respect to $\boldsymbol{B}_{0}$; Gary et al. 1976), kinetic instability driven by ion beams (Xiang et al. 2018), the losscone distribution for the electrons and ions (Ahirwar et al. 2006; Hua et al. 2011), and the gyrating pickup ions (Russell \& Blancocano 2007). Unlike LH-EMWs, RH-EMWs can be generated or excited by an opposite proton temperature anisotropy $\left(T_{\perp p}<T_{\| p}\right.$; Gary et al. 1976; Podesta \& Gary 2011) or relative drift between different ion populations (Gary 1985; Daughton \& Gary 1998). The abovementioned instabilities tend to suggest that the main excitation mechanism of EMWs around the proton cyclotron frequency is proton temperature anisotropy, and ion beams could be additional free energy that sustains the wave growth in the solar wind (Jian et al. 2016).

EMWs have been observed extensively in many plasma environments, such as the magnetosphere (Russell \& Blancocano 2007; Meredith et al. 2014; Sugiyama et al. 2015; Lee \& Lee 2016), the magnetotail (Nakagawa et al. 2018), the Earth's magnetosheath (Denton et al. 1994, 1998; Song et al. 1994), and the solar wind (Jian et al. 2009, 2010, 2014; Aggarwal et al. 2016; Gary et al. 2016; Wicks et al. 2016; Zhao et al. 2017a). In the solar wind, interplanetary magnetic clouds (IMCs) are the most important subset of CMEs erupted from the Sun and have a large-scale magnetic flux rope structure. The main observational signatures of IMCs are a low proton temperature, a low plasma beta, an enhanced magnetic field strength, and a smooth rotation of the magnetic field direction through a relatively large angle during a long time interval (about one day at 1 au; Burlaga et al. 1981, 1982; Klein \& Burlaga 1982; Marubashi 1986; Lepping et al. 1990; Schwenn \& Marsch 1991; Lynch et al. 2003; Owens et al. 2005). Furthermore, recent observations show that EMWs can extensively appear not only within IMCs but also in some complex structures around IMCs (Siu-Tapia et al. 2015; Zhao et al. 2017b). When an IMC moves at a velocity relative to the solar wind faster than the fast-mode wave speed, a driven shock can develop and a sheath region is formed and bounded by the IMC-driven shock and IMC-leading edge (Tsurutani et al. 1988; Liu et al. 2006; Feng et al. 2010; Guo et al. 2011). Different from the plasma environment inside an IMC, the sheath region usually has a higher proton temperature, higher plasma density, and higher plasma beta, as well as strong EMW fluctuations due to the interaction between the driven shock and 
Observation Parameters of IMC from STEREO-B

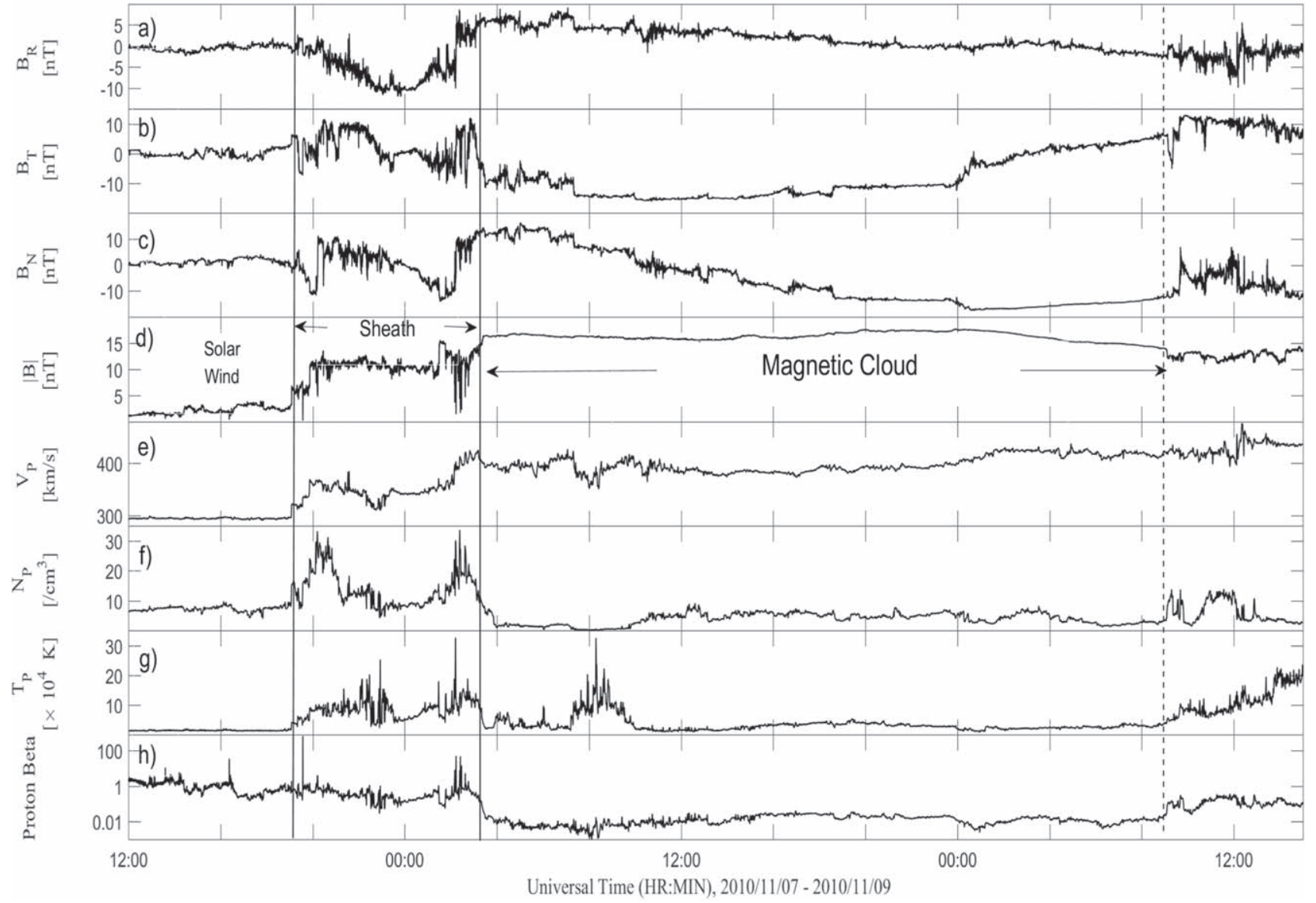

Figure 1. Overview of the magnetic field and plasma data for an IMC and its sheath region observed by STEREO-B during 2010 November 7-9 : (a)-(c) the components of the magnetic field $B_{R}, B_{T}, B_{N}$, respectively, (d) the magnetic field magnitude $|B|$, (e) the proton speed $V_{p}$, (f) the proton number density $N_{p}$, (g) the proton temperature $T_{p}$, and (h) proton beta. The first vertical solid line marks the time of the forward shock driven by the subsequent IMC. The second vertical solid line marks the leading edge of the IMC, and the vertical dashed line marks the ending edge of the IMC. The sheath region is between the two vertical solid lines.

the leading edge of the IMC. Thus, the sheath regions can be considered as a natural laboratory to study EMWs and their properties.

However, as far as we know, there has been relatively little analysis of EMWs in the sheath regions of IMCs in the solar wind. In this study we conduct a preliminary statistical analysis of EMWs in six IMC's sheath regions observed by the STEREO spacecraft (A and B). Based on the Morlet wavelet dynamic spectral wave analysis and the filter analysis, polarization senses of these EMWs are determined in the spacecraft and plasma frames. These results are helpful for us to better understand the physics of EMWs and the related wave-particle interaction.

The rest of this paper is organized as follows. The data and analysis methods are introduced in Section 2, and four typical EMW events are provided in detail in Section 3. Based on 81 EMW events, the statistical results in Section 4 are presented for the wave occurrence rates, local plasma characteristics, and wave properties in the spacecraft and plasma frames. Finally, Section 5 is devoted to related discussions and conclusions.

\section{Data and Methods}

In the present study, we use the $8 \mathrm{~Hz}$ magnetic field data and the $1 \mathrm{~min}$ plasma data from the In situ Measurements of Particles and CME Transients (Luhmann et al. 2008) and the
Plasma and Suprathermal Ion Composition (PLASTIC) instruments (Galvin et al. 2008) on board the STEREO twin spacecraft (A and B), respectively. Figure 1 provides an example of IMC observed by STEREO-B during 2010 November 7-9, and from top to bottom, shows the three components of the magnetic field $B_{R}, B_{T}, B_{N}$ in the radialtangential-normal (RTN) coordinate (where $\boldsymbol{R}$ points away from the Sun's center through the spacecraft, $\boldsymbol{N}$ is the projection of the solar rotation axis on the plane of the sky, and $\boldsymbol{T}$ is formed by the cross product of $\boldsymbol{N}$ and $\boldsymbol{R}$ and lies in the solar equatorial plane), the magnetic field magnitude $|B|$, the proton speed $V_{p}$, the proton number density $N_{p}$, the proton temperature $T_{p}$, and the proton beta, respectively. A forward shock driven by the IMC is observed at 19:05 UT on 2010 November 7, corresponding to the first solid vertical line in Figure 1. For this IMC, it starts at 02:46 UT on 2010 November 8 and ends at 09:46 UT on 2010 November 9. The interval enclosed by the two solid vertical lines in Figure 1 corresponds to the sheath region driven by the subsequent magnetic cloud. In total, six sheath regions of IMCs are chosen for further wave analysis in this paper. The plasma properties in the six sheath regions are shown in Table 1. Magnetic field and plasma parameters shown from column 5 to column 10 are average values inside the sheath regions. Column 10 of Table 1 shows that the average values of plasma $\beta$ are larger than 1 , except one event without available data. In addition, column 11 shows 


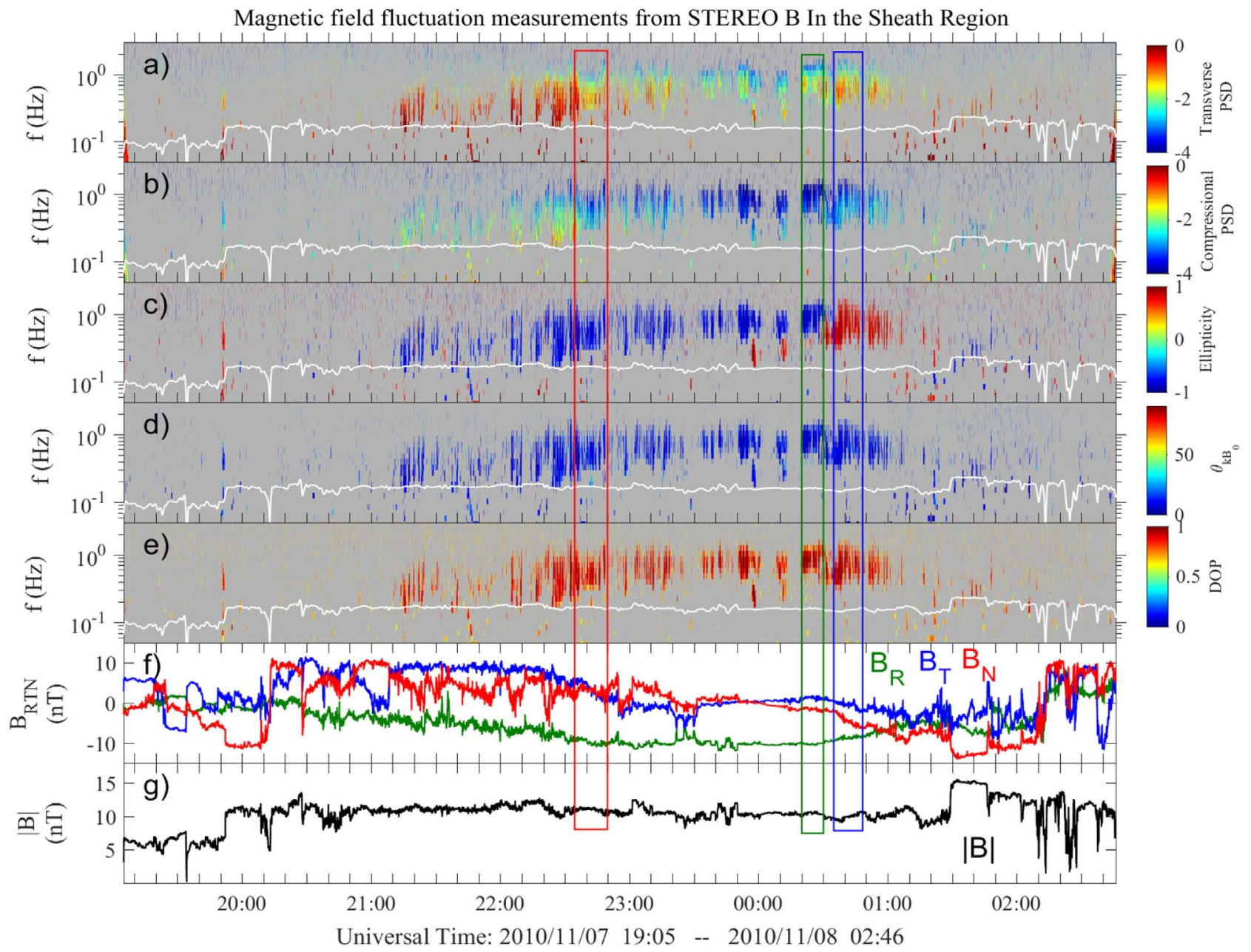

Figure 2. Magnetic field and its dynamic spectra in the sheath region of an IMC on 2010 November 7: (a) the transverse PSD, (b) the compressional PSD, (c) the ellipticity in the spacecraft frame (where positive (negative) sign corresponds to RH(LH)-circular polarization), (d) $\theta_{\boldsymbol{k} \boldsymbol{B}_{0}}$, (e) DOP, (f) magnetic field components in RTN coordinate, and $(\mathrm{g})$ the magnetic field magnitude $|B|$.

Table 1

Properties of the Selected Sheath Regions of IMCs

\begin{tabular}{|c|c|c|c|c|c|c|c|c|c|c|}
\hline $\begin{array}{l}\text { Shock } \\
\text { (UT) }\end{array}$ & $\begin{array}{l}\text { IMC Start }^{\mathrm{a}} \\
\text { (UT ) }\end{array}$ & $\begin{array}{l}\mathrm{IMC} \mathrm{End}^{\mathrm{b}} \\
\text { (UT) }\end{array}$ & $\mathrm{ST}^{\mathrm{c}}$ & $\begin{array}{l}|B| \\
\text { (nT) }\end{array}$ & $\begin{array}{c}N_{p} \\
\left(/ \mathrm{cm}^{3}\right)\end{array}$ & $\begin{array}{c}T_{p} \\
\left(\times 10^{4} \mathrm{~K}\right)\end{array}$ & $\begin{array}{c}V_{p} \\
\left(\mathrm{~km} \mathrm{~s}^{-1}\right)\end{array}$ & $\begin{array}{c}P_{t}^{\mathrm{d}} \\
(\mathrm{pPa})\end{array}$ & Plasma $\beta$ & $\begin{array}{l}\text { Pct. }^{\mathrm{e}} \\
(\%)\end{array}$ \\
\hline 2008 Jun 6 15:36 & 2008 Jun 6 22:15 & 2008 Jun 7 12:32 & $B$ & 9.14 & 15.11 & 8.35 & 401.75 & 84.44 & 1.87 & 4.8 \\
\hline 2011 Jun 16 21:00 & 2011 Jun 17 08:40 & 2011 Jun 18 06:00 & $B$ & 12.94 & 17.51 & 17.82 & 565.02 & 157.67 & 1.46 & 10.12 \\
\hline 2011 Sep 21 01:27 & 2011 Sep 21 06:14 & 2011 Sep 21 11:40 & $B$ & 6.89 & 6.81 & 6.56 & 375.05 & 39.7 & 1.11 & 5.94 \\
\hline 2009 Jan 24 17:20 & 2009 Jan 25 18:22 & 2009 Jan 27 10:00 & $B$ & 5.49 & 8.68 & 4.26 & 377.22 & 35.17 & 2.77 & 3.19 \\
\hline 2011 May 6 17:10 & 2011 May 6 22:00 & 2011 May 7 04:11 & $B$ & 5.98 & 6.02 & 7.43 & 440.19 & 34.78 & 1.98 & 22.13 \\
\hline
\end{tabular}

Notes.

a The start time of IMCs.

$\mathrm{b}$ The end time of IMCs.

c The STEREO spacecraft (A or B).

$\mathrm{d}$ The total pressure including thermal pressure and magnetic pressure.

e The duration ratio of the identified EMWs to the whole sheath region.

${ }^{\mathrm{f}}$ The sign $*$ indicates that no data are available.

the time duration ratio of the EMW activity to the entire sheath region from $3.2 \%$ to $24.3 \%$. From Figure 1 and Table 1, it is clear that the magnetic field and plasma parameters in the sheath region are highly perturbed and compressed due to the interaction between the solar wind and the IMC structure.
In order to select the candidate EMW events, the Morlet wavelet polarization analysis based on the singular value decomposition method (Santolík et al. 2003) is performed in the sheath region. From the identification criteria of Jian et al. (2009, 2010), the main characteristics of EMWs we studied 
here are as follows: first, the dominant component of the wave power is transverse to $\boldsymbol{B}_{0}$; second, these EMWs are nearly circularly polarized, and the corresponding absolute value of ellipticity $|\epsilon|$ is larger than 0.7 ( $\epsilon$, the ratio of axes of polarization ellipse, where the positive and negative signs indicate $\mathrm{RH}$ - and LH-polarized waves, respectively, see Means 1972 for details); third, the angle $\theta_{\boldsymbol{k} \boldsymbol{B}_{0}}$ between the wave vector $\boldsymbol{k}$ and the background magnetic field $\boldsymbol{B}_{0}$ is less than 20 degrees, implying that the waves are propagating quasiparallel to $\boldsymbol{B}_{0}$. Besides, the degree of polarization (DOP) should be greater than 0.7 to ensure a high signal-to-noise ratio.

Based on the criteria for identifying EMWs mentioned above, the dynamic magnetic filed spectrogram for the sheath region of the IMC in Figure 1 is presented in Figure 2. The area with both DOP and $|\epsilon|$ less than 0.7 is marked in gray. The wave fluctuations mainly occur between 21:00 UT and 01:30 UT. In this time interval, EMWs are intermittent with prolonged bursts lasting over one minute. In other regions, the EMWs appear sporadically with a short-duration of less than one minute. Comparing the transverse and compressional power spectral densities (PSDs) shown in Figures 2(a) and (b), it is clear that the transverse PSD is greater than the compressional one, satisfying the first criteria for identifying EMWs. As shown in Figures 2(c) and (d), the $|\epsilon|$ of EMWs is greater than 0.7, and the angle $\theta_{\boldsymbol{k} \boldsymbol{B}_{0}}$ is less than $20^{\circ}$. The wave features in Figure 2 clearly show that these EMWs are transverse, near-circularly polarized, and quasi-parallel propagating waves. In Figure 2, there are many gray intervals between any two consecutive EMW packets. If the gray interval is larger than the interval of either EMW packet, we will consider them as two EMW events. On the contrary, if the gray interval is far less than the duration of either EMW packet, we consider them as one wave event. It is important to emphasize that the analysis of each EMW should eliminate the effects of the magnetic structures, such as directional discontinuities. After a visual inspection of the magnetic field spectrogram, the positions and durations of the EMW events satisfying the above criteria will be selected for further wave analysis. The duration of every selected event in our study is longer than one minute, and those filamentous EMW events with a duration shorter than one minute will be ignored in this study.

To further explore the EMW properties in the plasma frame, the minimum variance analysis (MVA) is used to obtain three eigenvectors, and the corresponding minimum variance direction is parallel to $\boldsymbol{k}$. In the plasma frame, however, the polarization handedness of EMWs is independent of the sign of the wave propagation direction $\boldsymbol{k}$ (Stix 1962; Gary 1993; Gary et al. 2016). For circularly LH-polarized waves in the plasma frame, the maximum variance direction of wave field $i$ is nearly perpendicular to the $\boldsymbol{B}_{0}-\boldsymbol{k}$ plane. But for RH circularly polarized waves, $\boldsymbol{i}$ almost lies in the $\boldsymbol{B}_{0}-\boldsymbol{k}$ plane. Based on the different characteristics between LH and RH waves, two vectors $\boldsymbol{A}\left(=\frac{\boldsymbol{k} \times \boldsymbol{B}_{0}}{\left|\boldsymbol{k} \times \boldsymbol{B}_{0}\right|}\right)$ and $\boldsymbol{S}(=\boldsymbol{A} \times \boldsymbol{k})$ are introduced to build an orthogonal plasma frame (Stix 1962; Blanco-Cano \& Schwartz 1995; Jian et al. 2009, 2014). In this frame, if the observed angle $\theta_{A i}$ between $\boldsymbol{A}$ and $\boldsymbol{i}$ is smaller than $20^{\circ}$, the waves are considered LH-polarized. Similarly, if the observed angle $\theta_{S i}$ between $S$ and $\boldsymbol{i}$ is smaller than $20^{\circ}$, the waves are considered RH-polarized. In other words, the polarization handedness of EMWs in the plasma frame can be identified directly from the calculated values of $\theta_{A i}$ and $\theta_{S i}$.
It should be noted that the polarization handedness of EMWs in the plasma frame can be the same as or different from that in the spacecraft frame. Since the solar wind Alfvén Mach number is typically 5-8, and the EMW speed is of the order of the Alfvén speed $V_{\mathrm{A}}$ (Jian et al. 2009, 2010; Gary et al. 2016). The polarization sense the EMWs in the spacecraft frame depends on the wave propagation direction $\boldsymbol{k}$ with respect to the solar wind flow $\boldsymbol{V}_{\boldsymbol{p}}$ (Jian et al. 2009; Gary et al. 2016). By comparing the handedness of wave polarization between the spacecraft and plasma frames, the direction of $\boldsymbol{k}$ can be determined (Gary et al. 2016). When $\boldsymbol{k}$ is parallel to $\boldsymbol{V}_{\boldsymbol{p}}$, the wave polarization handedness will be the same between the plasma and spacecraft frames, and these waves are outward-propagating away from the Sun. When $\boldsymbol{k}$ is antiparallel to $\boldsymbol{V}_{\boldsymbol{p}}$, the wave polarization handedness in the spacecraft frame will suffer a reversal in their intrinsic handedness in the plasma frame, and such waves are inward-propagating toward the Sun. Due to the Doppler effect of the solar wind, the observed wave frequency $f_{\mathrm{ws}}$ is much higher than the intrinsic frequency $f_{\mathrm{wp}}$ in the solar wind. In order to remove the Doppler effect, $f_{\mathrm{wp}}$ can be calculated through

$$
f_{\mathrm{ws}}=f_{\mathrm{wp}}+\frac{\boldsymbol{k} \cdot \boldsymbol{V}_{\boldsymbol{p}}}{2 \pi} \approx f_{\mathrm{wp}}\left(1+\frac{V_{p}}{V_{A}} \hat{\boldsymbol{k}} \cdot \hat{\boldsymbol{V}}_{\boldsymbol{p}}\right)
$$

given by Jian et al. (2009).

On the other hand, the EMW bandwidth between the maximum wave frequency $f_{\max }$ and the minimum wave frequency $f_{\min }$ can be roughly estimated from the magnetic field spectrogram. Then, we can adopt low-pass and high-pass filter analysis on the magnetic field data with $f_{\min }$ and $f_{\max }$ to obtain the background magnetic field $\boldsymbol{B}_{0}$ and the wave field $\delta \boldsymbol{B}$ (Tsurutani et al. 2013; Remya et al. 2014). It is worth noting that the magnetic field below $f_{\text {min }}$ has a significant impact on the direction $i$ of the wave field, causing a broad variation of $\theta_{A i}$ or $\theta_{S i}$ between $0^{\circ}$ and $90^{\circ}$. To avoid or reduce this effect, we perform repeated filtering to get suitable values of $f_{\max }$ and $f_{\min }$ and then obtain $\boldsymbol{B}_{0}$ and $\delta \boldsymbol{B}$ more accurately.

\section{Case Studies}

After the procedure introduced in Section 2, 81 EMW events are found in the sheath regions driven by six IMCs. These EMWs can occur throughout the sheath regions. In the plasma frame, these EMWs are LH- or RH-polarized waves with magnetic perturbations transverse and propagating quasiparallel to $\boldsymbol{B}_{0}$. However, in the spacecraft frame, the polarization handedness of the waves will remain the same or be reversed depending on the direction of wave vector $\boldsymbol{k}$ relative to the solar wind velocity $\boldsymbol{V}_{\boldsymbol{p}}$. In this section, four typical EMW examples will be presented in detail.

\subsection{Case 1: Outward-propagating LH-EMW}

Figure 3 shows an example of an outward-propagating LHEMW event, where the wave vector $\boldsymbol{k}$ is oriented away from the Sun. This event occurred in the middle sheath region on 2010 November 7, corresponding to the region marked by the red box in Figure 2. Figures 3(a) and (b) indicate that the transverse PSD is dominant over the compressional one. Figures 3(c) and (d) show that for these waves the related ellipticity is lower than -0.7 and $\theta_{\boldsymbol{k} \boldsymbol{B}_{0}}$ is below $10^{\circ}$, which implies that the waves are LH-polarized and quasi-parallel propagating in the spacecraft frame. For this event, the time 
STEREO-B 2010-11-07 UTC, Polarization Analysis
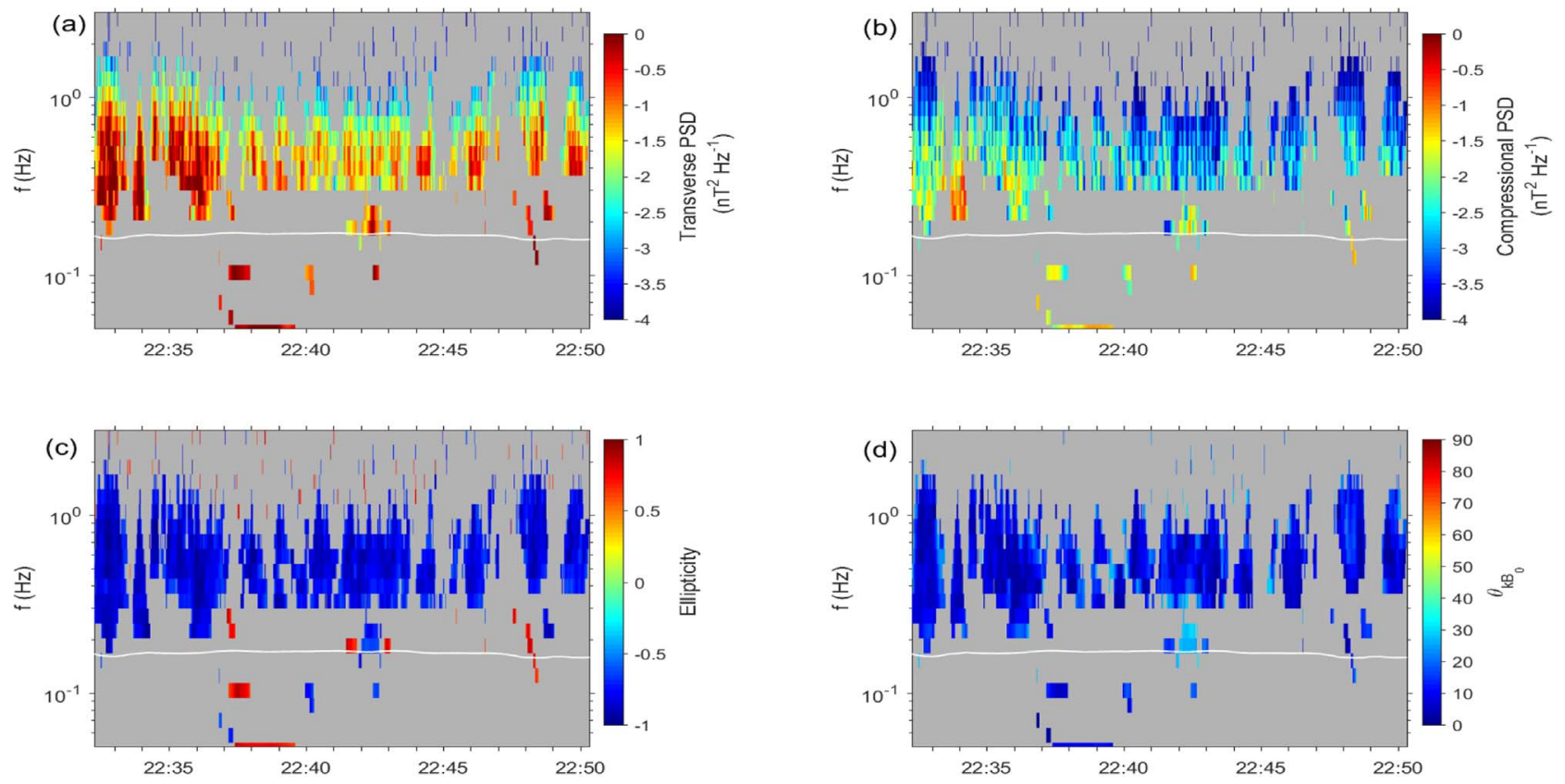

Figure 3. Dynamic spectra in the spacecraft frame for an outward-propagating LH-EMW event inside a fraction of the sheath region marked with the red box shown in Figure 2: (a) the transverse PSD, (b) the compressional PSD, (c) the ellipticity $\epsilon$, and (d) $\theta_{\boldsymbol{k} \boldsymbol{B}_{0}}$. Gray regions correspond to the signal with both DOP and $|\epsilon|$ below 0.7 . White traces indicate the local proton gyrofrequency.
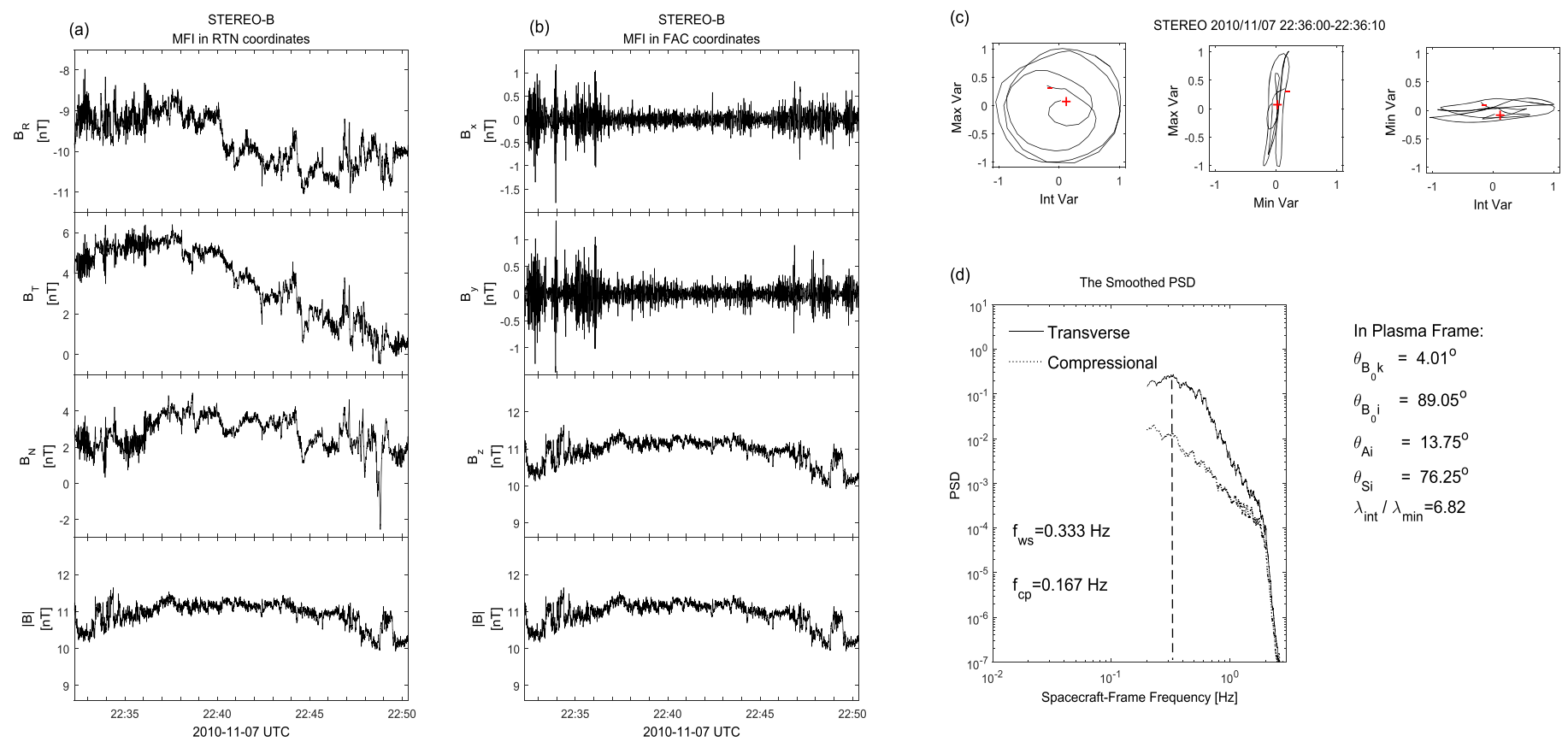

$$
\begin{aligned}
& \text { In Plasma Frame: } \\
& \theta_{\mathrm{B}_{0} \mathrm{k}}=4.01^{\circ} \\
& \theta_{\mathrm{B}_{0} \mathrm{i}}=89.05^{\circ} \\
& \theta_{\mathrm{Ai}}=13.75^{\circ} \\
& \theta_{\mathrm{Si}}=76.25^{\circ} \\
& \lambda_{\text {int }} / \lambda_{\min }=6.82
\end{aligned}
$$

Figure 4. Characteristics of outward-propagating LH-EMWs corresponding to the same time interval shown in Figure 3: (a) magnetic field data in RTN coordinate system, (b) magnetic field data in field-aligned coordinate system (FAC), (c) hodograms of the wave magnetic field from MVA, and (d) the PSDs of transverse and compressional magnetic fluctuations. The beginning and the ending of wave cycles are marked with + and - , respectively. In the first panel of Figure $4(\mathrm{c}), \boldsymbol{B}_{0}$ points to the paper.

duration is approximately 20 minutes from 22:32:16 UT to 22:50:20 UT. White traces in Figure 3 represent the local proton cyclotron frequency $f_{\mathrm{cp}}$ in the plasma frame. The components of the magnetic field in RTN coordinates are shown in Figure 4(a). In the field-aligned coordinate (FAC) system (Figure 4(b)), $\mathrm{z}$ component aligns with $\boldsymbol{B}_{0}$, and $x$ and $y$ components are perpendicular to $\boldsymbol{B}_{0}$. Both Figures 4(a) and (b) display clearly that the amplitude of the magnetic perturbation in the forequarter is more violent than the other parts. These characteristics are in agreement with the results of the polarization analysis in Figure 3.

After repeated filtering, we find that the suitable $f_{\min }$ and $f_{\max }$ values are $0.2 \mathrm{~Hz}$ and $1.8 \mathrm{~Hz}$, respectively, and consider the filtered magnetic field between $f_{\min }$ and $f_{\max }$ as the perturbed 
STEREO-B 2010-11-08 UTC, Polarization Analysis
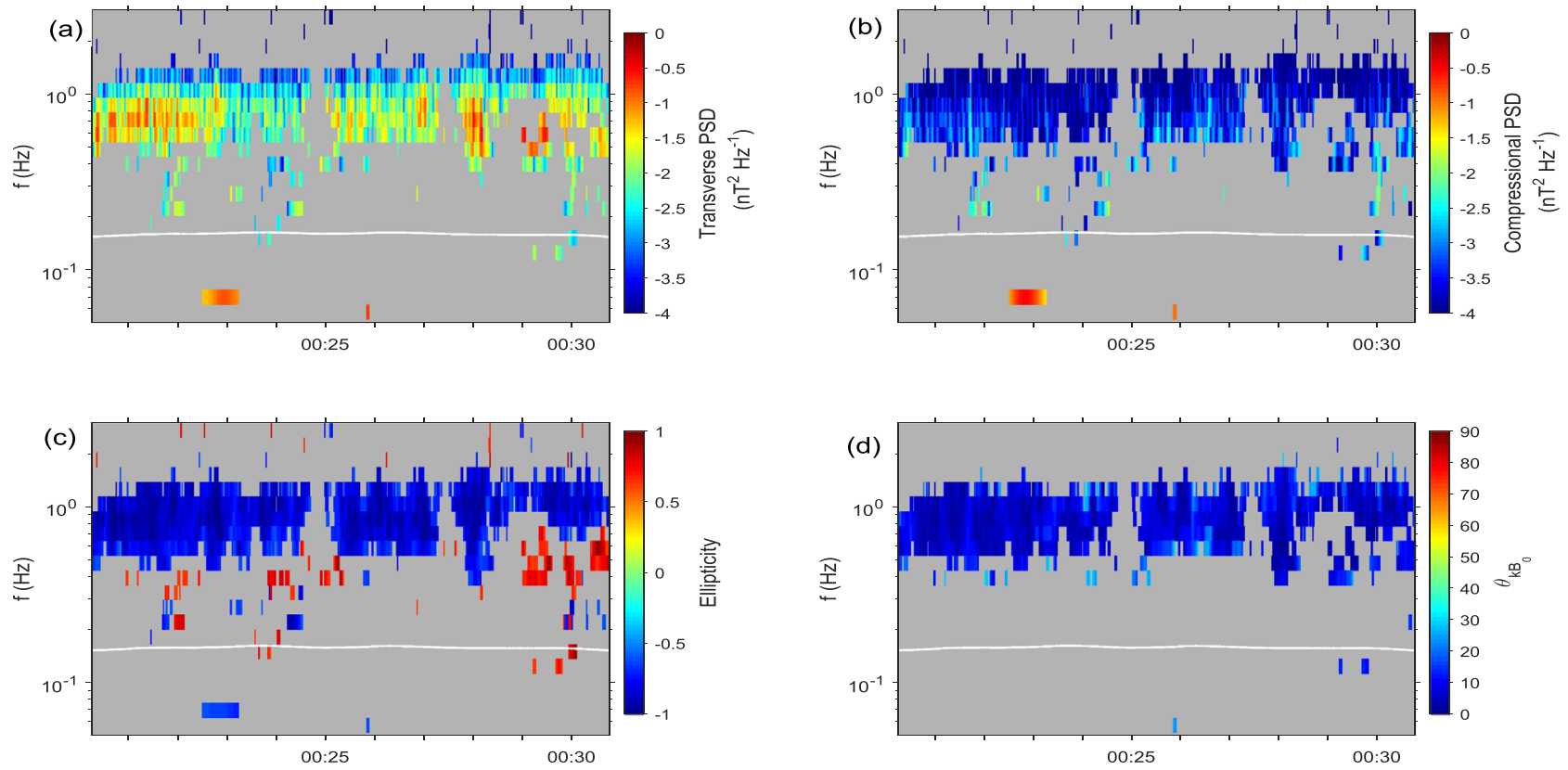

Figure 5. Dynamic spectra in the spacecraft frame for an inward-propagating RH-EMW event inside a fraction of the sheath region marked with the green box shown in Figure 2. The format is the same as that of Figure 3.

magnetic field $\delta \boldsymbol{B}$, and the difference between $\boldsymbol{B}$ and $\delta \boldsymbol{B}$ as the background magnetic field $\boldsymbol{B}_{0}$. Then, the wave vector $\boldsymbol{k}$ will be obtained by the MVA analysis of $\delta \boldsymbol{B}$. From the hodogram of $\delta \boldsymbol{B}$ in Figure 4(c), it can be seen that these waves are LH-polarized in the spacecraft frame and propagate quasi-parallel to $\boldsymbol{B}_{0}$ $\left(\theta_{\boldsymbol{k} \boldsymbol{B}_{0}} \approx 4^{\circ} .01\right)$, and the direction of $\boldsymbol{B}_{0}$ points into the paper. We also calculated the transverse and compressional PSDs of $\delta \boldsymbol{B}$ through a fast Fourier transform. For the transverse PSD in Figure 4(d), there is one peak frequency in the spacecraft frame: $f_{w s}=0.333 \mathrm{~Hz}$ (indicated by the vertical dashed line).

We further analyzed the polarization property of this event in the plasma frame. The angle $\theta_{A i}$ between the maximum variance direction $\boldsymbol{i}$ and the vector $\boldsymbol{A}$ is equal to 13.75 , while the angle $\theta_{S i}$ between $\boldsymbol{i}$ and the vector $\boldsymbol{S}$ is 76.25. This means that for these EMWs $\boldsymbol{i}$ is nearly perpendicular to the $\boldsymbol{B}_{0}-\boldsymbol{k}$ plane, that is to say, the polarization sense of the EMWs is intrinsically LH-polarized in the solar wind. From the spacecraft to plasma frames, the polarization handedness of these EMWs does not change. So we can deduce that the waves in this event are outward-propagating LH-EMWs relative to the solar wind velocity $\boldsymbol{V}_{\boldsymbol{p}}$ (Gary et al. 2016). Using the Doppler shift relation of Equation (1), the wave frequency in the plasma frame is $f_{\mathrm{wp}} \approx 0.0678 \mathrm{~Hz}$, below the local proton cyclotron frequency $f_{\mathrm{cp}}(=0.167 \mathrm{~Hz})$.

\subsection{Case 2: Inward-propagating RH-EMW}

An example for the polarization reversal of LH-EMWs in the spacecraft frame is presented in the same sheath region marked by the green box in Figure 2. In this interval, the dynamic spectra in Figures 5(a) and (b) indicate that these EMWs have a strong transverse component. The ellipticity in Figure 5(c) shows that these EMWs are LH-polarized waves in the spacecraft frame. The wave propagation direction is nearly quasi-parallel to $\boldsymbol{B}_{0}$, because $\theta_{\boldsymbol{k} \boldsymbol{B}_{0}}$ for these waves is below $10^{\circ}$ (Figure $5(\mathrm{~d})$ ). The corresponding DOP is larger than 0.7 . Figures 6(a) and (b) show the magnetic field characteristics of these LH-EMWs in the RTN and FAC coordinates. Based on the bandwidth range of the wave in Figure 5, the suitable $f_{\text {min }}$ and $f_{\max }$ values can be obtained by repeated filtering, i.e., $f_{\min }=0.4 \mathrm{~Hz}$ and $f_{\max }=1.3 \mathrm{~Hz}$. Then we perform the MVA of the wave field. In Figure 6(c), the hodogram of the wave magnetic field clearly indicates that these EMWs are LHpolarized in the spacecraft frame.

Then, we analyzed the wave polarization sense in the plasma frame. The angle $\theta_{\boldsymbol{k} \boldsymbol{B}_{0}}$ between the wave vector $\boldsymbol{k}$ and the background magnetic field $\boldsymbol{B}_{0}$ is about $2^{\circ}$, but the angle $\theta_{\boldsymbol{A} i}$ is $76^{\circ} .2$ and the angle $\theta_{S i}$ is approximately equal to $14^{\circ}$. It implies that the maximum-perturbation direction $\boldsymbol{i}$ of these waves is nearly parallel to the $\boldsymbol{B}_{0}-\boldsymbol{k}$ plane. Based on the results above, it can be inferred that these LH-EMWs observed in the spacecraft frame are intrinsically inward-propagating RH-EMWs in the solar wind (Siu-Tapia et al. 2015; Gary et al. 2016). Figure 6(d) shows the smoothed Fourier spectra of the wave field data in the entire region. There is a peak frequency $\left(f_{\mathrm{ws}}=0.705 \mathrm{~Hz}\right.$ in the spacecraft frame) in the transverse component. After removing the Doppler effect via Equation (1), the peak frequency would be $f_{\mathrm{wp}}=0.205 \mathrm{~Hz}$ in the plasma frame, above the local proton cyclotron frequency $f_{\mathrm{cp}}(=0.16 \mathrm{~Hz})$. It is very possible that these RH-EMWs are essentially magnetosonic/whistler waves propagating toward the Sun in the plasma frame.

\subsection{Case 3: Outward-propagating RH-EMW}

There also exists a region where the ellipticity is greater than 0.7 (the blue box illustrated in Figure 2). Figure 7 displays the dynamic spectra for this region from 00:31:00 UT to 00:49:20 UT on 2010 November 8 . The polarization characteristics in this region indicate that these EMWs are RH-polarized with a frequency above the local proton cyclotron frequency in the spacecraft frame. We use the same wave analysis as in cases 1 and 2 in order to determine the polarization sense of these EMWs in the plasma frame. Based on the bandwidth range of 

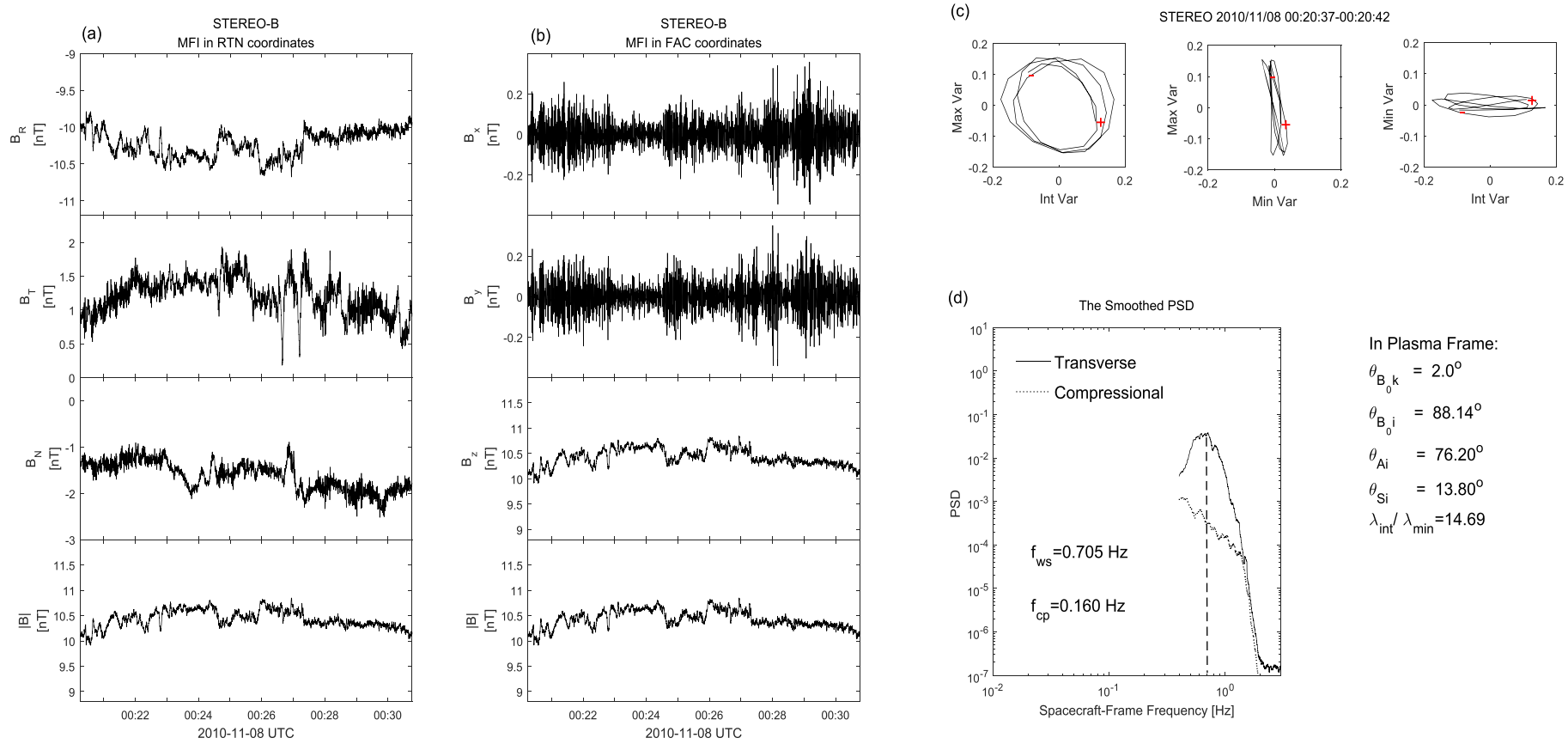

$$
\begin{aligned}
& \text { In Plasma Frame: } \\
& \theta_{\mathrm{B}_{0} \mathrm{k}}=2.0^{\circ} \\
& \theta_{\mathrm{B}_{0} \mathrm{i}}=88.14^{\circ} \\
& \theta_{\mathrm{Ai}}=76.20^{\circ} \\
& \theta_{\mathrm{Si}}=13.80^{\circ} \\
& \lambda_{\text {int }} / \lambda_{\min }=14.69
\end{aligned}
$$

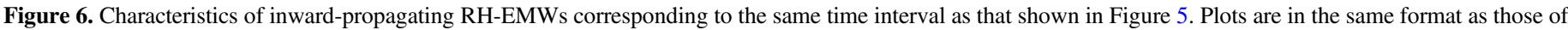
Figure 4.

STEREO-B 2010-11-08 UCT, Polarization Analysis
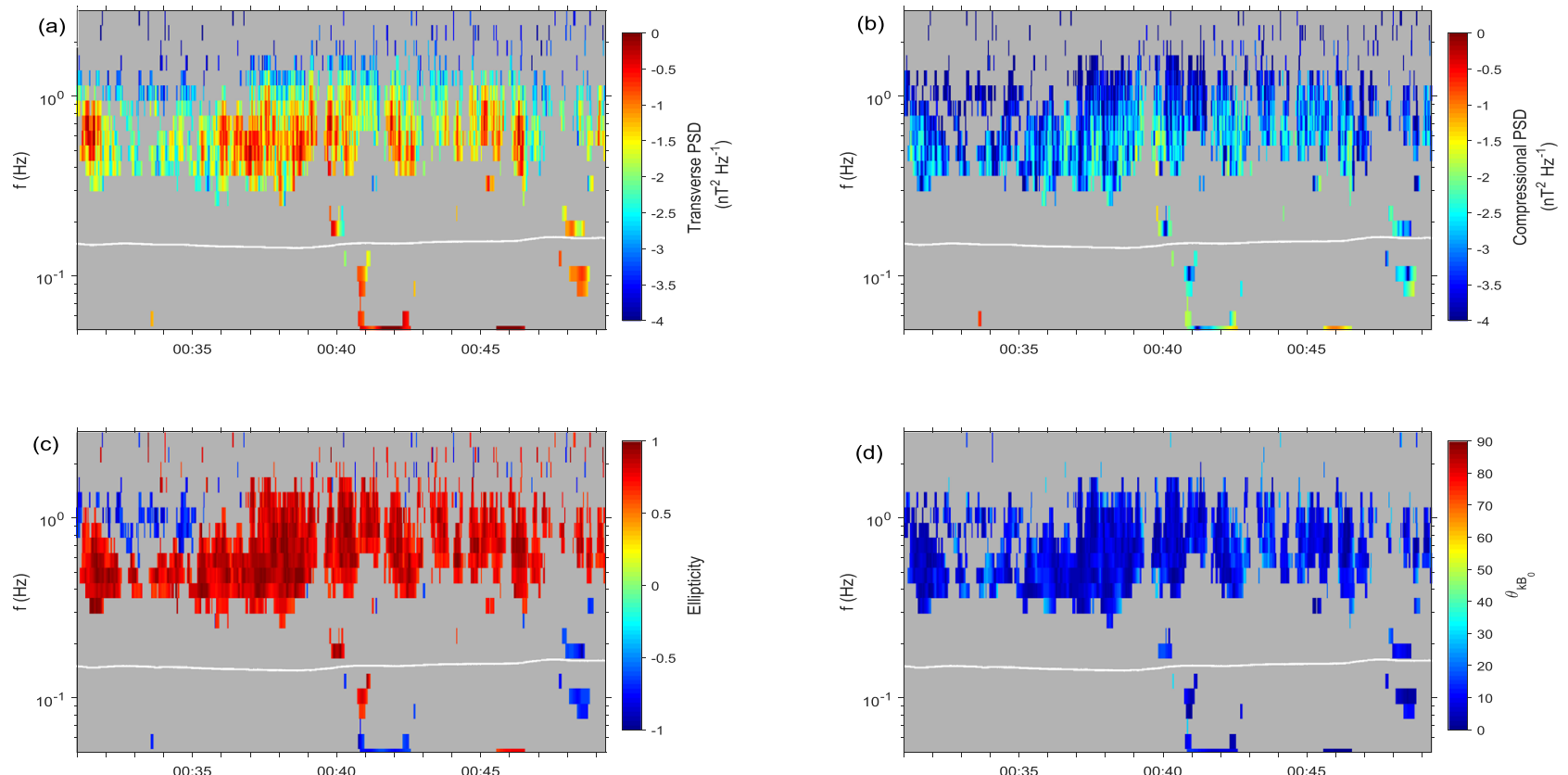

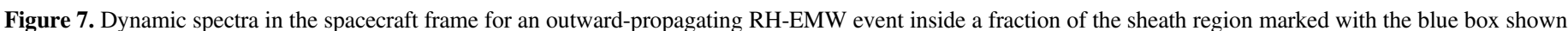
in Figure 2. The format is the same as that of Figure 3.

the waves in Figure 7, we perform repeated filtering to get suitable $f_{\min }$ and $f_{\max }$, i.e., $0.3 \mathrm{~Hz}$ and $1.8 \mathrm{~Hz}$, respectively. Then, $\delta \boldsymbol{B}$ and $\boldsymbol{B}_{0}$ can be obtained. The MVA results in Figure $8(\mathrm{c})$ show that these EMWs are RH circularly polarized and propagating quasi-parallel to $\boldsymbol{B}_{0}$ due to $\theta_{\boldsymbol{k} \boldsymbol{B}_{0}} \approx 1^{\circ}$, in agreement with the polarization analysis in Figure 7(c). Because the angle $\theta_{S i}=18^{\circ} .7$ is lower than $20^{\circ}$ in the plasma frame, it can be deduced that the maximum variance direction $i$ of the wave magnetic field lies in the $\boldsymbol{B}_{0}-\boldsymbol{k}$ plane. So these EMWs can be considered as RH-polarized EMWs in the plasma frame. From the polarization handedness in the spacecraft and plasma frames, it is possible to conclude that these RH-polarized waves are outward-propagating RH-EMWs in the solar wind. On the other hand, the transverse Fourier power in Figure $8(\mathrm{~d})$ reveals a peak frequency $f_{\mathrm{ws}}=0.548 \mathrm{~Hz}$ in the spacecraft frame. Using the Doppler shift relation 

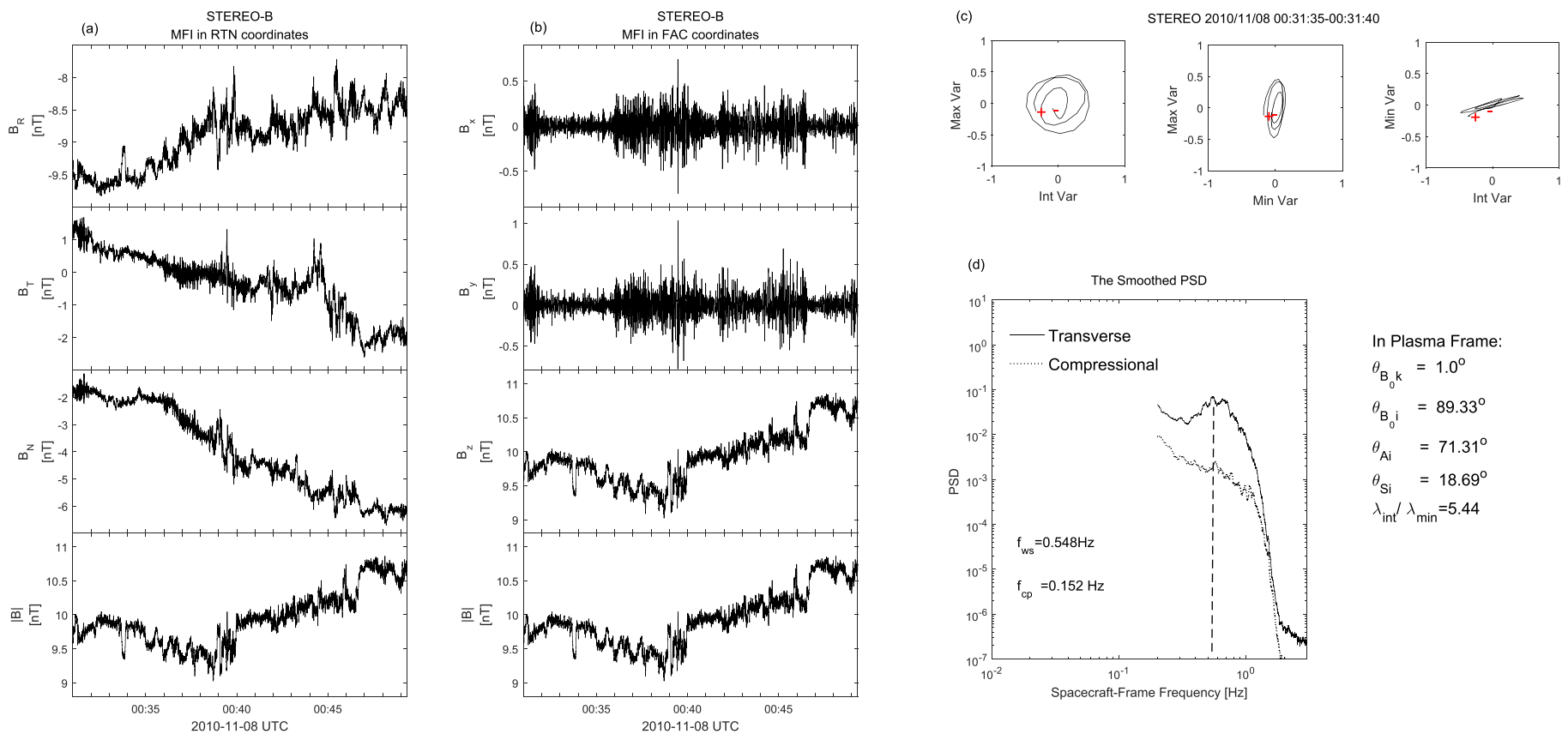

$$
\begin{aligned}
& \text { In Plasma Frame: } \\
& \theta_{\mathrm{B}_{0} \mathrm{k}}=1.0^{\circ} \\
& \theta_{\mathrm{B}_{0} \mathrm{i}}=89.33^{\circ} \\
& \theta_{\mathrm{Ai}}=71.31^{\circ} \\
& \theta_{\mathrm{Si}}=18.69^{\circ} \\
& \lambda_{\mathrm{int}} / \lambda_{\min }=5.44
\end{aligned}
$$

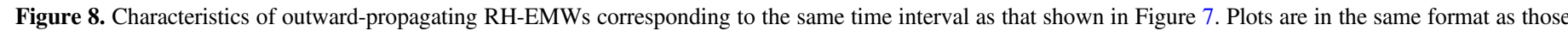
in Figure 4.

STEREO-A 2011-05-06 UCT, Polarization Analysis
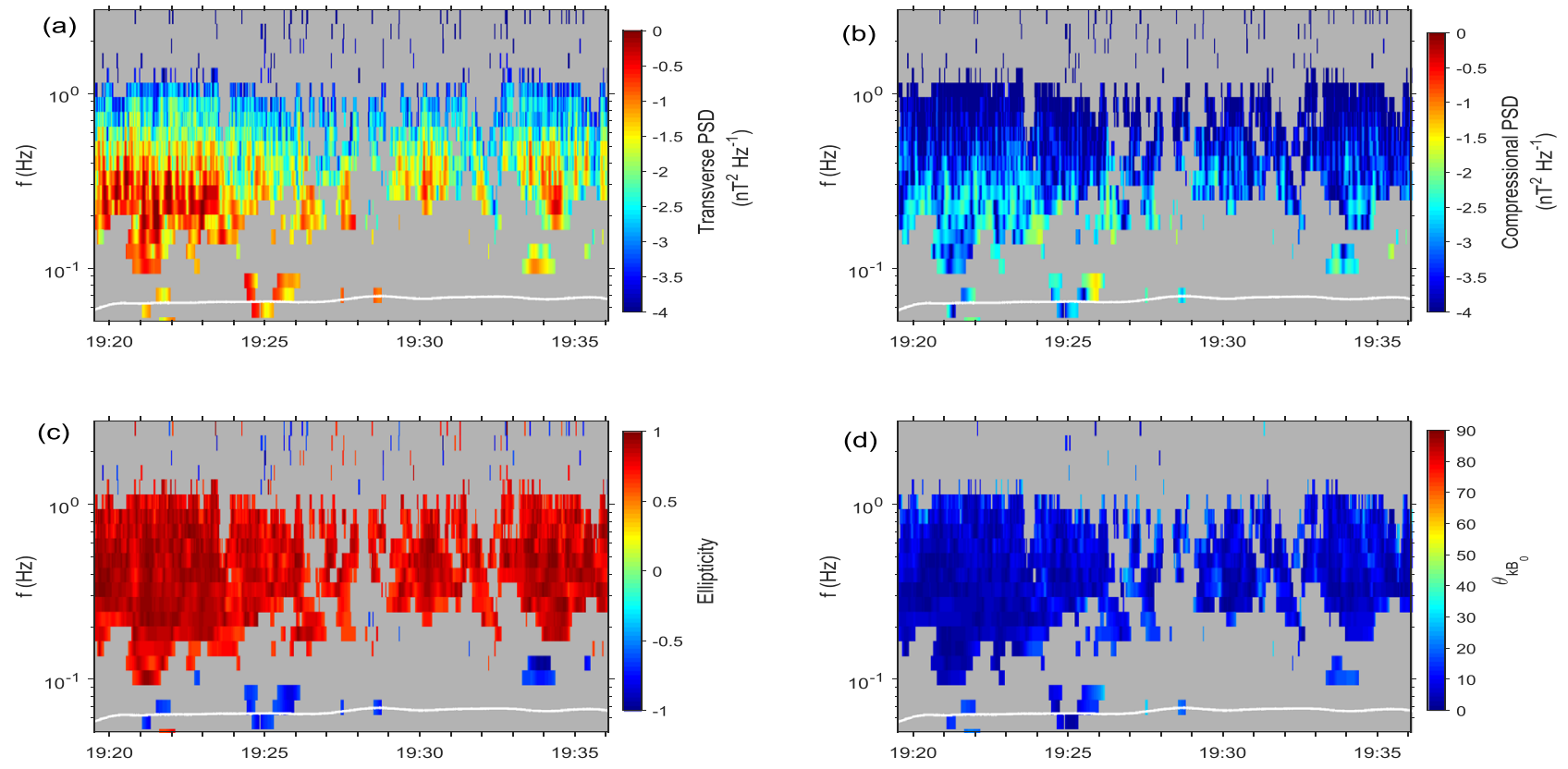

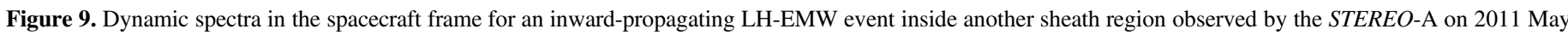
6. The format is the same as that of Figure 3.

(Equation (1)), the wave frequency in the plasma frame can be calculated: $f_{\mathrm{wp}}=0.108 \mathrm{~Hz}$, close to the local proton cyclotron frequency $f_{\text {cp }}=0.151 \mathrm{~Hz}$.

\subsection{Case 4: Inward-propagating LH-EMW}

Figures 9 and 10 illustrate an inward-propagating LH-EMW event, which takes place in an IMC-driven sheath observed by STEREO-A on 2011 May 6. These inward-propagating waves lasted about 16.6 minutes. Comparing the spectra in Figures 9(a) and (b), the transverse PSD is much larger than the compressional one, especially on both sides of this interval. The ellipticity value is above 0.7 (Figure 9(c)), and the propagation angle $\theta_{\boldsymbol{k} \boldsymbol{B}_{\mathbf{0}}}$ is below $10^{\circ}$ (Figure 9(d)). These characteristics above suggest that these EMWs are circularly RH-polarized waves in the spacecraft frame, and propagate quasi-parallel to the background magnetic field. The hodogram of the wave field $\delta \boldsymbol{B}$ in MVA coordinates are plotted in Figure $10(\mathrm{c})$, where the magnetic field perturbation is $\mathrm{RH}$ around $\boldsymbol{B}_{0}$ (out of the paper), consistent with the results of the polarization analysis in Figure 9(c). The transverse and 

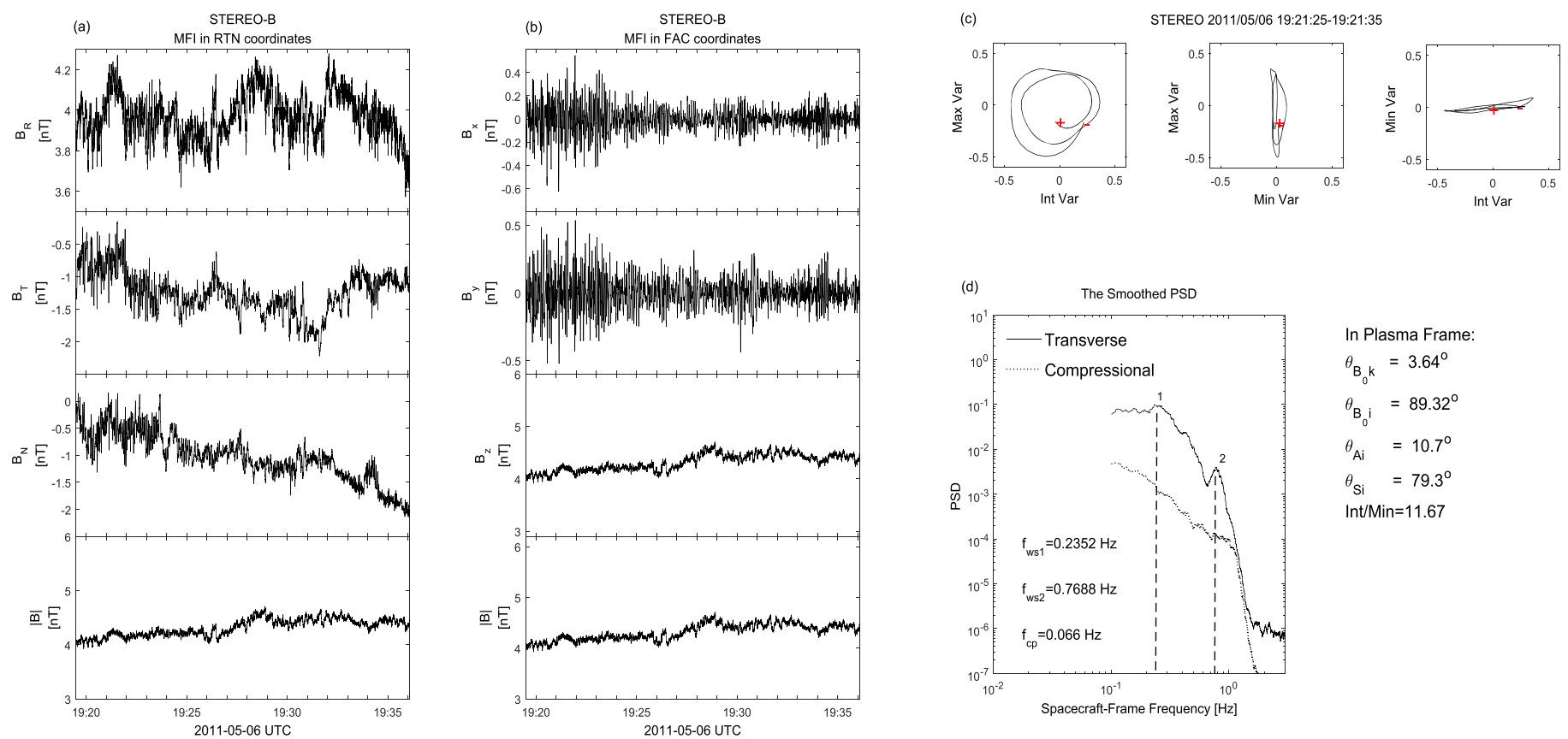

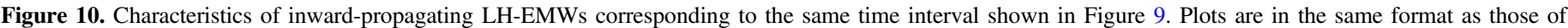
Figure 4.

compressional PSDs in the spacecraft frame are shown in Figure 10(d), in which there are two peak frequencies in the transverse component: $f_{\mathrm{ws} 1}=0.235 \mathrm{~Hz}$ and $f_{\mathrm{ws} 2}=0.769 \mathrm{~Hz}$.

In the plasma frame, however, the angles $\theta_{A i}$ and $\theta_{S i}$ based on the MVA results are $10^{\circ} .7$ and $79^{\circ} .3$, respectively, which means that the EMWs are LH-polarized. These EMWs suffer a polarization reversal from the spacecraft to plasma frames. Thus, we can conclude that these RH-polarized EMWs in the spacecraft frame are inward-propagating LH-EMWs in the plasma frame (Gary et al. 2016). Accordingly, the two peak frequencies in the plasma frame are, respectively, $f_{\mathrm{wp} 1}=0.022 \mathrm{~Hz}$ and $f_{\mathrm{wp} 2}=0.071 \mathrm{~Hz}$, below or near the local proton cyclotron frequency $\left(f_{\mathrm{cp}}=0.066 \mathrm{~Hz}\right)$.

\section{Results}

Based on the STEREO observations and the methods introduced in Section 2, a total of 81 EMW events are examined in six selected sheath regions of IMCs, accounting for $9.2 \%$ of the total analysis time. The percentage of the total duration for the identified EMWs over the time of the entire sheath region is shown in Table 1 . The duration of these waves can be from 60 to $1225 \mathrm{~s}$, with a median of $139 \mathrm{~s}$. The MVA results of the wave field indicate that the wave events with the ratio $\lambda_{\text {int }} / \lambda_{\min }$ of the intermediate to minimum eigenvalues greater than 5 occupy $86.4 \%$ of all EMW events, that is, most selected wave events are planar waves. The polarization sense of these EMWs can be LH- or RH-polarized in the spacecraft frame. Moreover, the majority of the EMWs in this study have one peak frequency in the power spectra, except seven events with two peak frequencies. Detailed statistical properties of EMWs within the sheath regions will be introduced from the following two aspects: the spacecraft and plasma frames.

In the spacecraft frame, 45 events (55.6\% of all events) are LHpolarized, slightly larger than the number of RH-polarized EMWs. Figure 11 displays the histogram comparison of the average values of various parameters between these LH- (dashed) and
RH-EMWs (solid) in the spacecraft frame. The vertical lines denote the median values of corresponding parameters, where the dashed and solid lines are for LH- and RH-EMWs, respectively. Both LH- and RH-EMWs have similar occurrence rate distributions on the proton density (Figure 11(a)), the proton temperature (Figure 11(b)), the total pressure (Figure 11(d)), the wave normalized amplitude (Figure 11(e)), the propagation angle (Figure 11(f)), the proton beta (Figure 11(g)), and the wave frequency (Figure 11(k)). Most EMWs have propagation angles $\theta_{\boldsymbol{k} \boldsymbol{B}_{0}}$ less than $10^{\circ}$ (see Figure 11(f)), implying that the majority of the waves are propagating quasi-parallel to the background magnetic field in the sheath regions. As shown in Figure 11(c), in a slow proton speed condition $\left(<400 \mathrm{~km} \mathrm{~s}^{-1}\right)$, the occurrence rate of the LH-EMWs is considerably higher than that of the RHEMWs. While in a high proton speed condition $\left(>550 \mathrm{~km} \mathrm{~s}^{-1}\right)$, the occurrence rate of the RH-EMWs is higher than that of LHEMWs. The mean normalized amplitudes $\delta B / B_{0}$ for the LH- and RH-EMWs (Figure 11(e)) are all less than 0.1. The occurrence rate of the logarithmic proton beta $\beta_{p}$ reveals that most EMWs are observed in low- $\beta_{p}(<1)$ regions (Figure 11(g)). In the case of $0.1<\beta_{p}<1$, the occurrence rate of $\mathrm{LH}$ waves is larger than that of $\mathrm{RH}$ waves. But when $\beta_{p}<0.1$, the occurrence rate of $\mathrm{RH}$ waves is greater than that of LH waves. In addition, the occurrence rates of the normalized minimum and maximum wave frequencies are given in Figures 11(h) and (i). From Figure 11(j) we can see that both LH- and RH-EMWs have a similar distribution for the normalized wave bandwidth. Comparing the median values between LH- and RH-EMWs in the spacecraft frame shown in Figure 11, we have some statistical results as follows. The median values of the proton density, temperature, velocity, and total pressure of RH waves are always larger than those of LH waves. In contrast with RH waves, $\mathrm{LH}$ waves have a slightly higher $f_{\mathrm{ws}}$ and broader bandwidth in the sheath regions, which are different from the observations of EMWs inside IMCs (Siu-Tapia et al. 2015; Zhao et al. 2017b). For example, the median $f_{\text {ws }}$ for LH and RH waves is $0.267 \mathrm{~Hz}$ and $0.235 \mathrm{~Hz}$, respectively (see Figure $11(\mathrm{k})$ ). The median value of the 


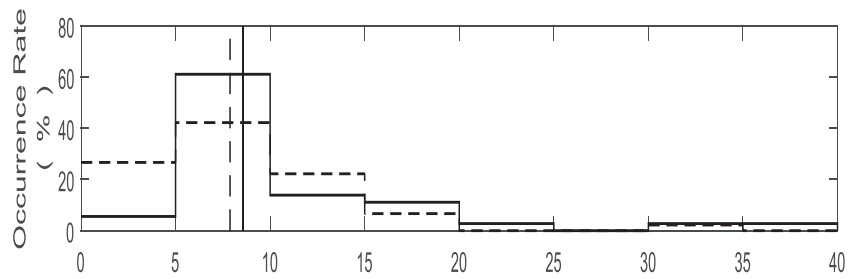

(a) $\mathrm{N}_{\mathrm{p}}\left(/ \mathrm{cm}^{-3}\right)$

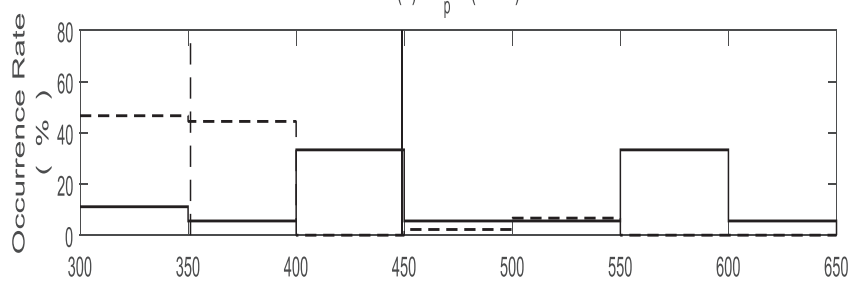

(c) $V_{p}(\mathrm{~km} / \mathrm{s})$

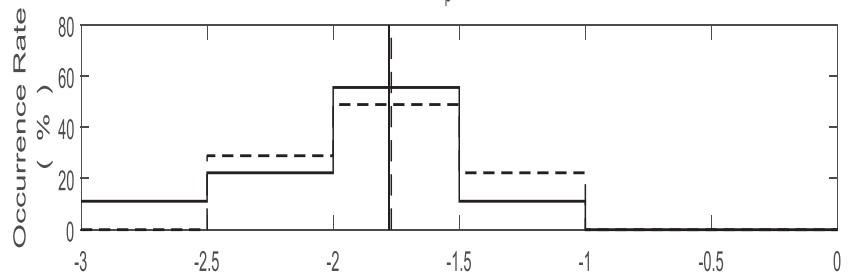

(e) $\log _{10} \delta B / B$
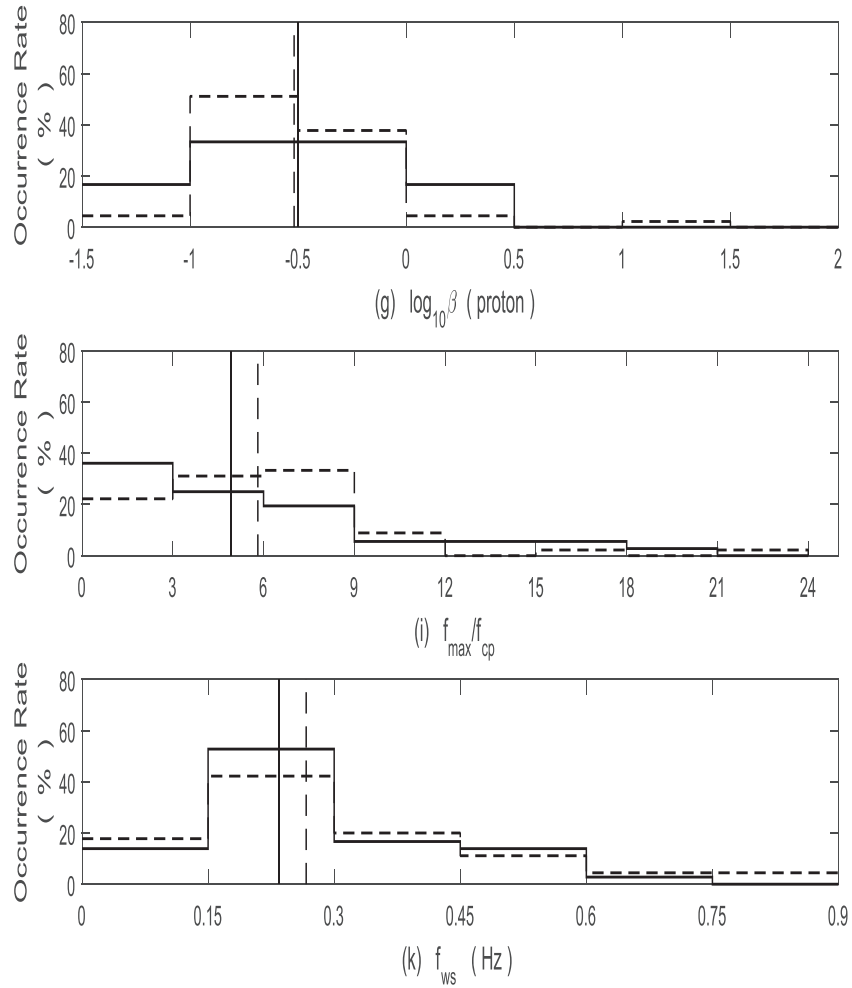

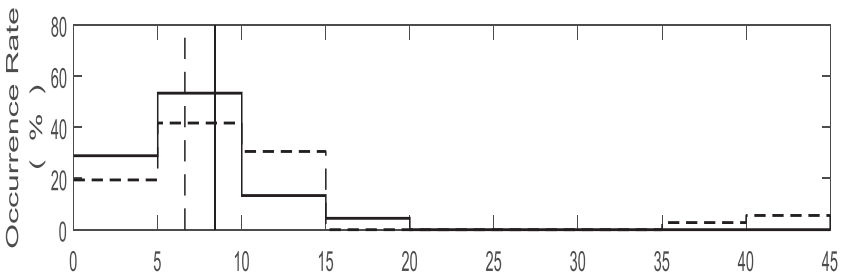

(b) $T_{p}\left({ }^{*} 10^{4} K\right)$
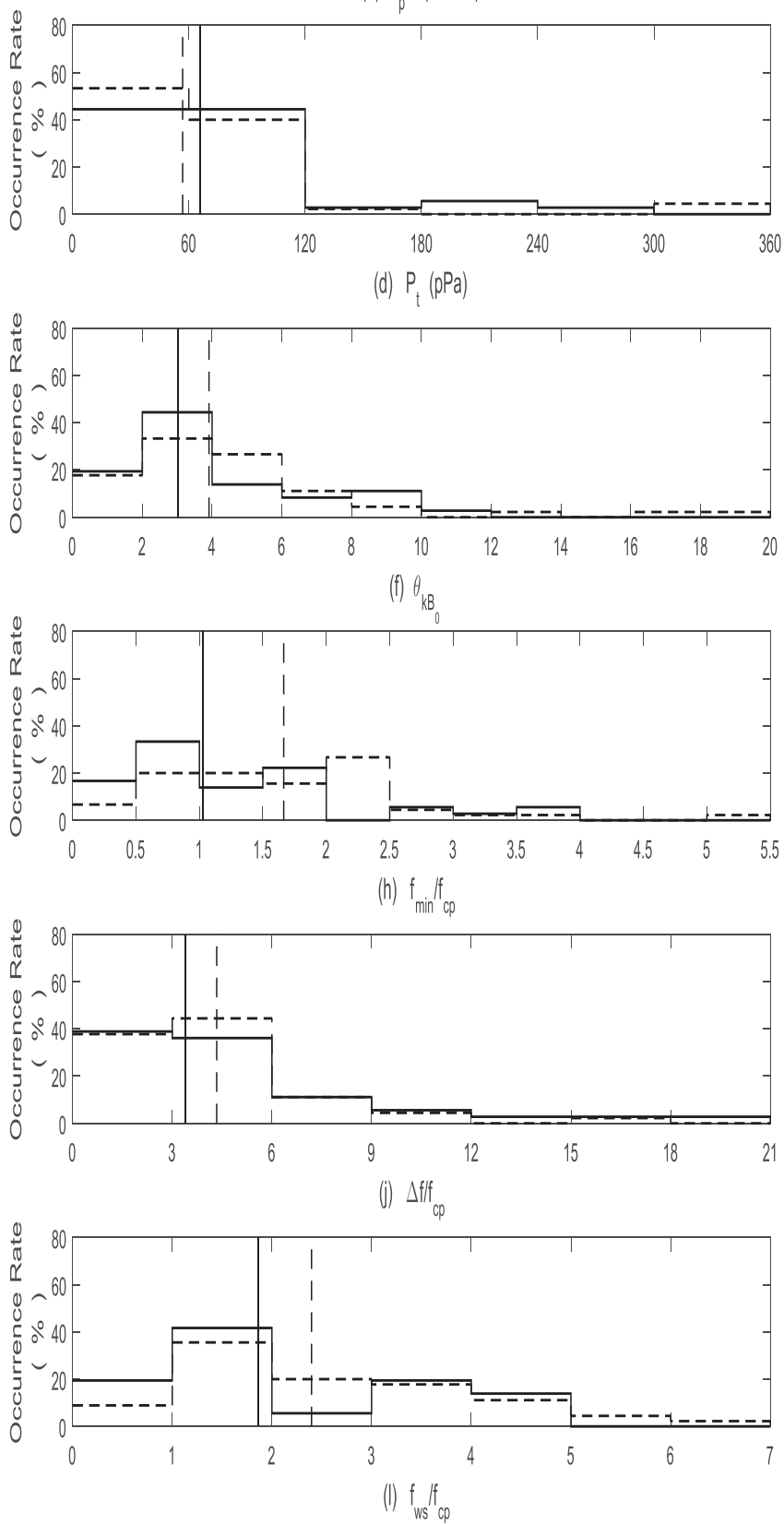

Figure 11. Histogram comparison of wave characteristics between LH- (dashed) and RH-EMWs (solid) in the spacecraft frame. Occurrence rates of (a) the proton density $N_{p}$, (b) proton temperature $T_{p}$, (c) proton velocity $V_{p}$, (d) total pressure $P_{t}$, (e) normalized wave amplitude $\delta B / B_{0}$, (f) propagation angle $\theta_{\boldsymbol{k} \boldsymbol{B}_{0}}$, (g) proton beta $\beta$, (h) normalized minimum wave frequency $f_{\min } / f_{\mathrm{cp}}$, (i) normalized maximum wave frequency $f_{\max } / f_{\mathrm{cp}}$, (j) normalized wave bandwidth $\Delta f / f_{\mathrm{cp}}$, (k) wave frequency $f_{\mathrm{ws}}$, and (l) normalized wave frequency $f_{\mathrm{ws}} / f_{\mathrm{cp}}$. The vertical dashed (solid) line indicates the median values of LH (RH) wave parameters.

normalized $\Delta f / f_{\text {cp }}$ for LH and RH waves in Figure 11(j) is 4.339 and 3.395 , respectively.

The polarization senses of these EMWs are also analyzed in the plasma frame. Different from the results in the spacecraft frame, the occurrence rate of RH-EMWs is slightly higher than that of LH-polarized EMWs, occupying 55.6\% of all events in the plasma frame. The histogram comparison of plasma and wave parameters is shown in Figure 12 with the same format as in Figure 11. The distributions of plasma parameters of LH and RH waves are similar, except that $\mathrm{RH}$ waves have a broader 


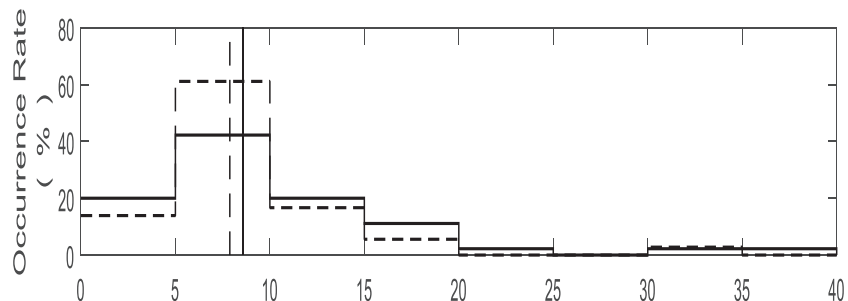

(a) $N_{0}\left(\left(\mathrm{~cm}^{3}\right)\right.$

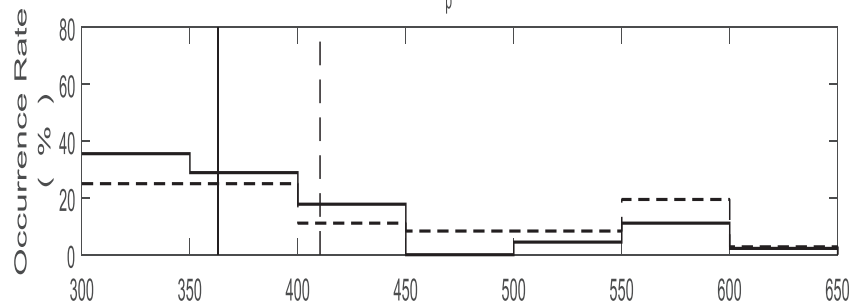

(c) $V_{p}(\mathrm{~km} / \mathrm{s})$

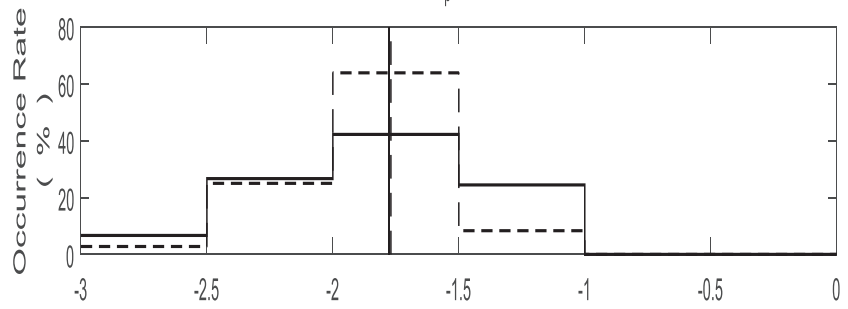

(e) $\log _{10} 8 B B_{0}$
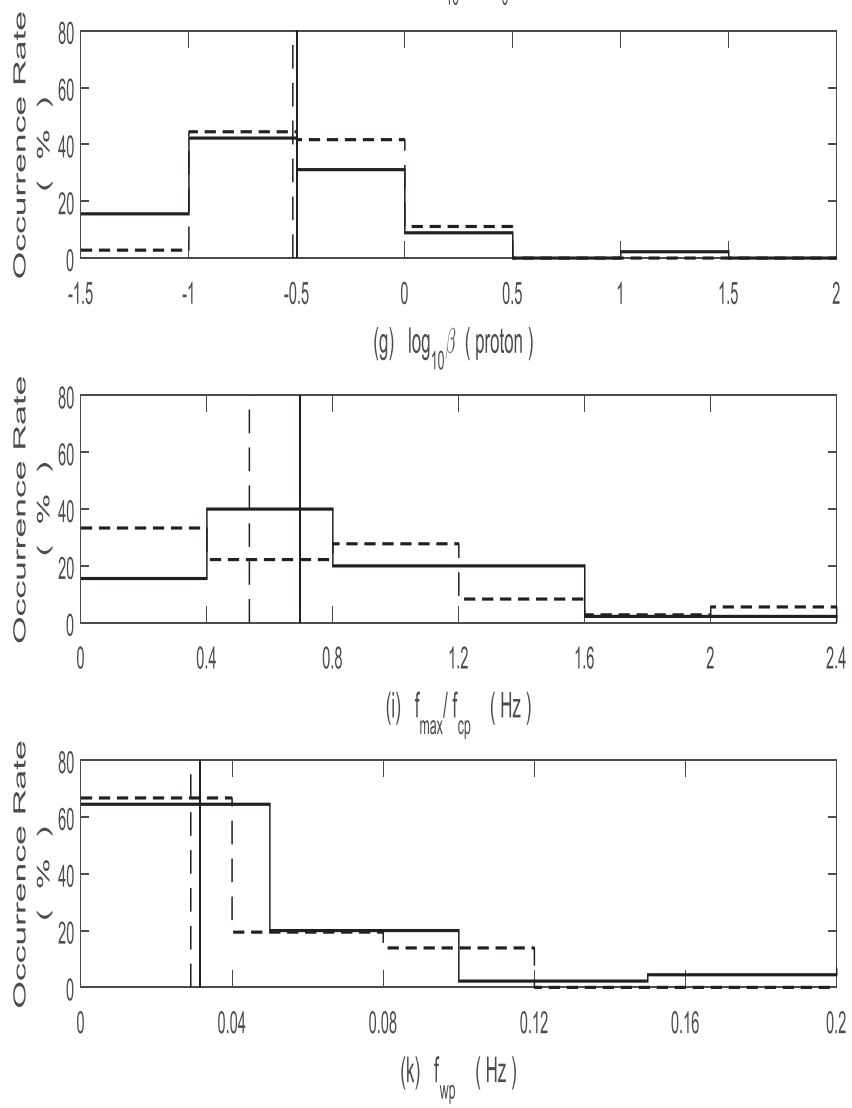

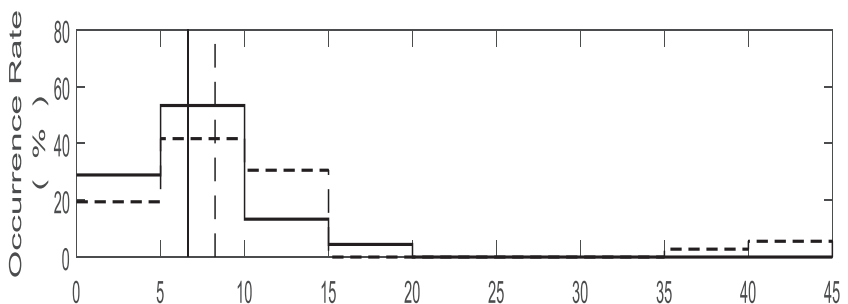

(b) $\left.T_{p}{ }^{(10} 0^{4} K\right)$

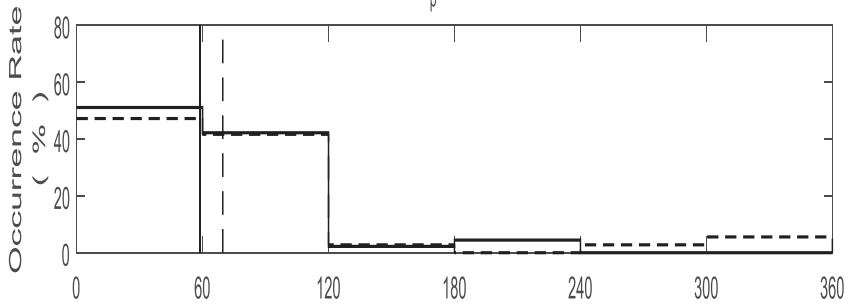

(d) $P_{1}(\mathrm{pPa})$
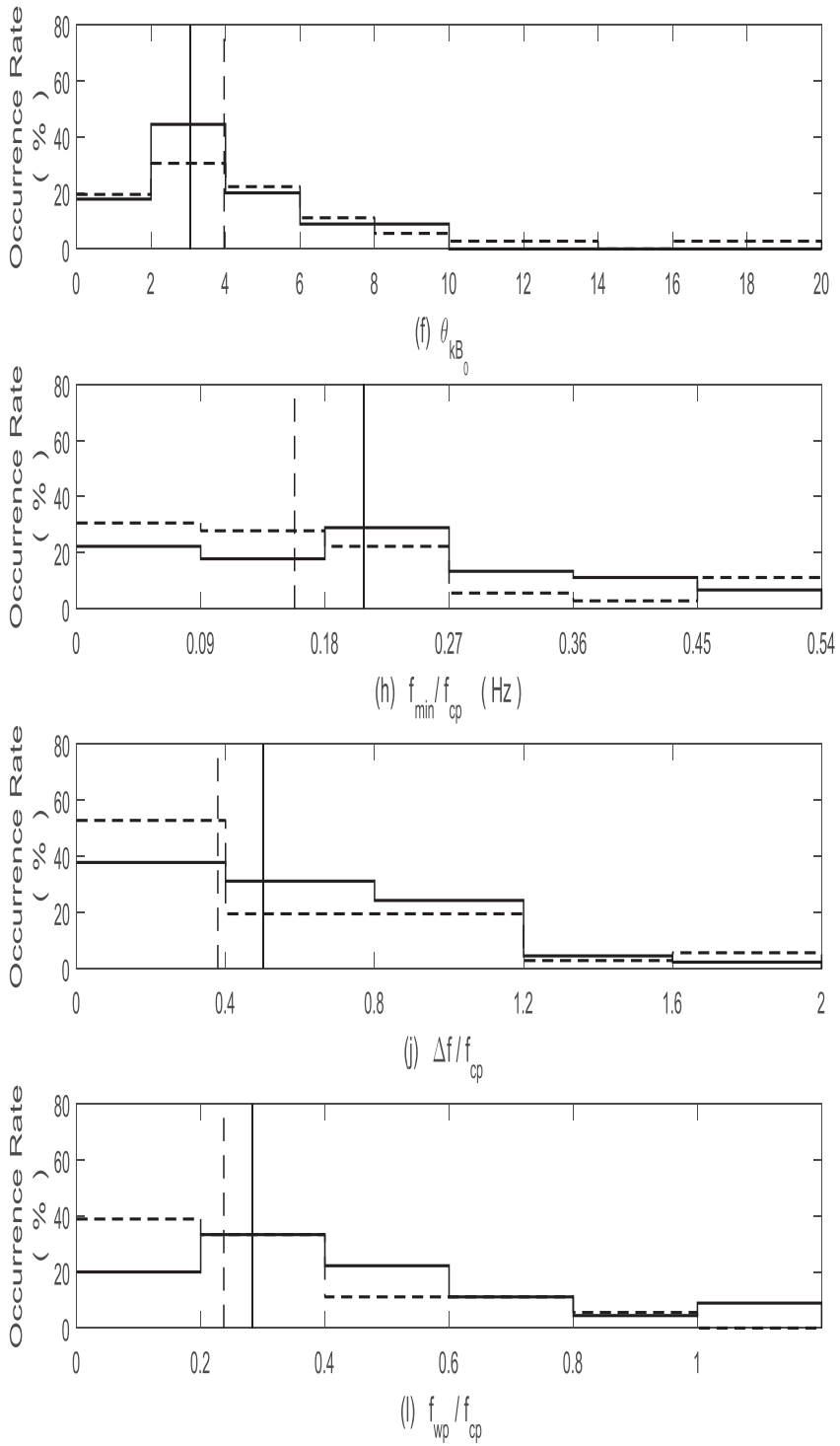

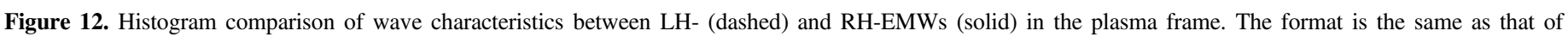
Figure 11. 
bandwidth, slightly higher $f_{\mathrm{wp}}$, and lower proton velocity in the plasma frame. In intermediate- and high-speed sheath regions, the occurrence rates of $\mathrm{LH}$ waves are larger than those of $\mathrm{RH}$ waves (Figure 12(c)). The propagation angles $\theta_{\boldsymbol{k} \boldsymbol{B}_{0}}$ of $\mathrm{RH}$ waves are all within $10^{\circ}$, but some LH waves have $10^{\circ}<\theta_{\boldsymbol{k} \boldsymbol{B}_{0}}<$ $20^{\circ}$ (Figure 12(f)). In the plasma frame, all LH waves and most $\mathrm{RH}$ waves are below the local proton cyclotron frequency. The median $f_{\mathrm{wp}} / f_{\text {cp }}$ is 0.29 for $\mathrm{LH}$ waves and 0.32 for $\mathrm{RH}$ waves (Figure 12(1)). From the spacecraft to plasma frames, more than half of the wave events in our study suffer a reversal of the polarization handedness in the sheath regions. Among these EMWs with polarization reversal, $19.75 \%$ are LH-EMWs and $30.86 \%$ are RH-EMWs in the plasma frame. These polarization-reversed EMWs are inward-propagating waves in the plasma frame (i.e., propagating toward the Sun). For LHEMWs in the sheath regions of IMCs, the maximum occurrence rate is found for the outward-propagating EMW events. But for RH-EMWs in the sheath regions, the maximum occurrence rate is found for the inward-propagating wave events. It is important that both outward-propagating LHEMWs and inward-propagating RH-EMWs around the proton cyclotron frequency are all circularly LH-polarized in the spacecraft frame due to the Doppler shift effect of the solar wind (Podesta \& Gary 2011; Gary et al. 2016). Similarly, both inward-propagating LH-EMWs and outward-propagating RHEMWs are all circularly RH-polarized in the spacecraft frame.

\section{Conclusion and Discussion}

EMWs around the proton cyclotron frequency $f_{\text {cp }}$ are very common in a variety of solar wind plasma environments and play an important role in related plasma heating and particle acceleration. The compressed and turbulent sheath regions of IMCs provide a natural environment for plasma instabilities to excite these EMWs. In this study, we performed a detailed analysis of such EMWs within the sheath regions of IMCs observed by the STEREO spacecraft. This is the first attempt to systematically analyze EMWs in the sheath regions of IMCs. Based on the Morlet wavelet spectral analysis and the repeated filtering method, 81 EMW events with a duration larger than one minute are identified to further study their plasma characteristics and wave properties (including the wave amplitude, duration, and bandwidths). All EMWs we studied here are transverse, circularly polarized, and parallel propagating waves, and have small amplitudes $\left(\delta B / B_{0}<0.1\right)$ throughout the sheath regions. The time duration of these EMWs can last from 1 to $\sim 19$ minutes with a median duration of $139 \mathrm{~s}$. In particular, the polarization sense of these EMWs is compared between the spacecraft and plasma frames. The polarization of these waves can be LH- or RH-polarized in the spacecraft frame. The occurrence rate of $\mathrm{LH}$ waves (55.6\% of all events) is slightly higher than that of RH waves, which is consistent with previous observations in the spacecraft frame (Jian et al. 2009, 2010, 2014; Zhao et al. 2017b). Our results also show that more than half of the wave events suffer a polarization reversal from the spacecraft to plasma frames, and as expected, all these polarization-reversed EMWs are inward-propagating EMWs relative to the solar wind flow in the sheath regions (Gary et al. 2016). Moreover, in the plasma frame, the outwardpropagating LH-EMWs and inward-propagating RH-EMWs have occurrence rates relatively higher than those of the inward-propagating LH-EMWs and outward-propagating $\mathrm{RH}$ EMWs, respectively.
Based on the detailed analysis of EMWs in the plasma frame, our results show that both LH- and RH-polarized EMWs can exist and propagate toward (i.e., inward-propagating) or away (i.e., outward-propagating) from the Sun in the sheath regions of IMCs. In general, the occurrence rates of RH-EMWs are slightly higher than those of LH-EMWs in the plasma frame, which is opposite to the results in the spacecraft frame. For inward-propagating EMWs, their polarization sense will suffer a reversal from the spacecraft to plasma frames (Gary et al. 2016). But for outward-propagating EMWs, the polarization sense will remain the same. As a result, the polarization sense of inward-propagating $\mathrm{RH}(\mathrm{LH})$-EMWs will be $\mathrm{LH}(\mathrm{RH})$ waves in the spacecraft frame. More than half of wave events suffer a polarization reversal, that is to say, such EMWs are inward-propagating waves in the solar wind. Among these inward waves in the plasma frame, 16 events are LH-EMWs and 25 events are RH-EMWs. For outwardpropagating EMWs, RH and LH waves have an equal number of wave events (20 events). Therefore, both outward-propagating LH-EMWs and inward-propagating RH-EMWs have a maximum occurrence rate in the sheath regions of IMCs, which is consistent with the theoretical predictions by Podesta \& Gary (2011). For these two wave modes, they show an LH-circular polarization with respect to $\boldsymbol{B}_{0}$ in the spacecraft frame. In the plasma frame, all the frequencies of LH-EMWs are below the local proton cyclotron frequency, but the frequencies of $\mathrm{RH}$ EMWs can exceed the local proton cyclotron frequency. For these reasons, we conclude that in the plasma frame all LHEMWs are ion cyclotron waves, and all RH-EMWs are magnetosonic/whistler waves.

There are multiple possible sources to excite EMWs within the sheath regions of IMCs. The sheath regions are often influenced by the IMC-driven shocks, which can provide effective free energy to excite EMWs (e.g., Liu et al. 2006). The plasma in the sheath regions is often characterized by a larger perpendicular temperature anisotropy $\left(T_{\perp p}>T_{\| p}\right)$, because the reflected ions gyrate back to the shock and enter the downstream region, producing plasma heating perpendicular to $\boldsymbol{B}_{0}$ (Crooker \& Siscoe 1977; Sckopke et al. 1983). Under such a temperature anisotropy condition, linear Vlasov theory predicts that LH-EMWs can be excited via ion cyclotron instability, especially in low- $\beta$ plasmas (Price et al. 1986; Fuselier et al. 1991; Gary et al. 1993; Lu \& Wang 2006). The related maximum growth rate of the cyclotron instability lies in the parallel direction, and the generated LH-EMWs propagate parallel to $\boldsymbol{B}_{0}$ (Gary 1992; Gary et al. 1993). Observational studies also show that the perpendicular temperature anisotropy of protons can be 1.2-1.3 in the sheath regions of IMCs (Liu et al. 2006), being a possible excitation mechanism to excite LH-EMWs. Two-dimensional hybrid simulations associated with fast shocks show that a bunch distribution for the helium ions in the perpendicular velocity phase space can excite strong EMWs (Lee 2017; Hao et al. 2014). On the other hand, in the hybrid simulations of upstream and downstream waves at the quasi-parallel shocks, Krauss-Varban \& Omidi (1991, 1993) and Krauss-Varban (1994) argued that the upstream waves driven by the right-handed ion beam instability can be mode converted into LH-EMWs in the downstream regions via the front shock. Another effective excitation mechanism for RHEMWs is the parallel firehose instability driven by parallel proton temperature anisotropy $\left(T_{\| p}>T_{\perp p}\right)$ (Gary 1993), which may not work in the sheath regions of IMCs, implying that RH- 
EMWs may be excited by some other mechanisms in the sheath regions or generated outside the sheath regions. For example, when the differential alpha-proton flow velocity and ion beams are taken into account, both LH- and RH-polarized EMWs around $f_{\mathrm{cp}}$ can be excited to propagate quasi-parallel to $\boldsymbol{B}_{0}$ (Podesta \& Gary 2011). Maybe EMWs are not generated by local mechanisms. The excitation source may be in the solar wind or the magnetic clouds, where many EMWs have been observed (Jian et al. 2009, 2010, 2014; Siu-Tapia et al. 2015; Gary et al. 2016; Zhao et al. 2017b). More work needs to be done to clarify the excitation mechanisms of EMWs in the sheath regions of IMCs.

This work was supported in part by the Specialized Research Fund for State Key Laboratories, NSFC under grants 41874201, 41531071, 11873018, 11790302, and 11761131007. We thank the STEREO/PLASTIC team and NASA's CDAWeb for making these data available. The authors also would like to thank Drs. L. Xiang, Y. F. Hao, and H. Y. Sun for their useful suggestions and discussions.

\section{ORCID iDs}

\section{J. Wu (1) https://orcid.org/0000-0001-8058-2765}

\section{References}

Aggarwal, P., Taylor, D. K., Smith, C. W., et al. 2016, ApJ, 822, 94 Ahirwar, G., Varma, P., \& Tiwari, M. S. 2006, AnGeo, 24, 1919 Blanco-Cano, X., \& Schwartz, S. J. 1995, AdSpR, 15, 97 Burlaga, L., Sittler, E., Mariani, F., \& Schwenn, R. 1981, JGR, 86, 6673 Burlaga, L. F., Klein, L., Sheeley, N. R., Jr., et al. 1982, GeoRL, 9, 1317 Crooker, N. U., \& Siscoe, G. L. 1977, JGR, 82, 185

Daughton, W., \& Gary, S. P. 1998, JGR, 103, 20613

Denton, R. E., Anderson, B. J., Gary, S. P., \& Fuselier, S. A. 1994, JGR, 99 11225

Denton, R. E., Lessard, M. R., LaBelle, J. W., \& Gary, S. P. 1998, JGR, 103, 23661

Feng, H. Q., Wu, D. J., Chao, J. K., Lee, L. C., \& Lyu, L. H. 2010, JGRA, 115 , A04107

Fuselier, S. A., Klumpar, D. M., Shelley, E. G., Anderson, B. J., \& Coates, A. J. 1991, JGR, 96, 21

Galvin, A. B., Kistler, L. M., Popecki, M. A., et al. 2008, SSRv, 136, 437

Gary, S. P. 1985, ApJ, 288, 342

Gary, S. P. 1992, JGR, 97, 8519

Gary, S. P. 1993, Theory of Space Plasma Microinstabilities (Cambridge: Cambridge Univ. Press)

Gary, S. P., Fuselier, S. A., \& Anderson, B. J. 1993, JGR, 98, 1481

Gary, S. P., Jian, L. K., Broiles, T. W., et al. 2016, JGRA, 121, 30
Gary, S. P., Montgomery, M. D., Feldman, W. C., \& Forslund, D. W. 1976, JGR, 81, 1241

Guo, J., Feng, X., Emery, B. A., et al. 2011, JGRA, 116, A05106

Hao, Y., Lu, Q., Gao, X., et al. 2014, JGRA, 119, 3225

Hollweg, J. V. 1975, RvGSP, 13, 263

Hua, Y. P., Wu, D. J., \& Wang, D. Y. 2011, ChSBu, 56, 257

Jian, L. K., Moya, P. S., Viñas, A. F., \& Stevens, M. 2016, in AIP Conf. Proc. 1720, Solar Wind 14, ed. K. T. Das, B. Kristiawan, \& M. S. Shekhawat (Melville, NY: AIP), 040007

Jian, L. K., Russell, C. T., Luhmann, J. G., et al. 2009, ApJL, 701, L105

Jian, L. K., Russell, C. T., Luhmann, J. G., et al. 2010, JGRA, 115, A12115

Jian, L. K., Wei, H. Y., Russell, C. T., et al. 2014, ApJ, 786, 123

Klein, L. W., \& Burlaga, L. F. 1982, JGR, 87, 613

Krauss-Varban, D. 1994, JGR, 99, 2537

Krauss-Varban, D., \& Omidi, N. 1991, JGR, 96, 17715

Krauss-Varban, D., \& Omidi, N. 1993, GeoRL, 20, 1007

Lee, K. H. 2017, JGRA, 122, 7307

Lee, K. H., \& Lee, L. C. 2016, GeoRL, 43, 9406

Lepping, R. P., Jones, J. A., \& Burlaga, L. F. 1990, JGR, 95, 11957

Liu, Y., Richardson, J. D., Belcher, J. W., Kasper, J. C., \& Skoug, R. M. 2006 JGRA, 111, A09108

Lu, Q. M., \& Wang, S. 2006, JGR, 111, A05204

Luhmann, J. G., Curtis, D. W., Schroeder, P., et al. 2008, SSRv, 136, 117

Lynch, B. J., Zurbuchen, T. H., Fisk, L. A., \& Antiochos, S. K. 2003, JGRA, 108, 1239

Marsch, E. 2006, LRSP, 3, 1

Marubashi, K. 1986, AdSpR, 6, 335

Means, J. D. 1972, JGR, 77, 5551

Meredith, N. P., Horne, R. B., Kersten, T., Fraser, B. J., \& Grew, R. S. 2014, JGRA, 119, 5328

Nakagawa, T., Nishino, M. N., Tsunakawa, H., et al. 2018, JGRA, 123, 1146

Omidi, N., Thorne, R., \& Bortnik, J. 2011, JGRA, 116, A09231

Owens, M. J., Cargill, P. J., Pagel, C., Siscoe, G. L., \& Crooker, N. U. 2005, JGRA, 110, A01105

Podesta, J. J., \& Gary, S. P. 2011, ApJ, 742, 41

Price, C. P., Swift, D. W., \& Lee, L.-C. 1986, JGR, 91, 101

Remya, B., Tsurutani, B. T., Reddy, R. V., et al. 2014, ApJ, 793, 6

Russell, C., \& Blancocano, X. 2007, JASTP, 69, 1723

Santolík, O., Parrot, M., \& Lefeuvre, F. 2003, RaSc, 38, 1010

Schwenn, R., \& Marsch, E. 1991, Physics of the Inner Heliosphere II. Particles, Waves and Turbulence, Physics and Chemistry in Space (Berlin: Springer)

Sckopke, N., Paschmann, G., Bame, S. J., Gosling, J. T., \& Russell, C. T. 1983, JGR, 88, 6121

Siu-Tapia, A., Blanco-Cano, X., Kajdic, P., et al. 2015, JGRA, 120, 2363

Song, P., Russell, C. T., \& Gary, S. P. 1994, JGR, 99, 6011

Stix, T. H. 1962, The Theory of Plasma Waves (New York: McGraw-Hill)

Sugiyama, H., Singh, S., Omura, Y., et al. 2015, JGRA, 120, 8426

Tsurutani, B. T., Echer, E., Richter, I., Koenders, C., \& Glassmeier, K.-H. 2013, P\&SS, 75, 17

Tsurutani, B. T., Gonzalez, W. D., Tang, F., Akasofu, S. I., \& Smith, E. J. 1988, JGR, 93, 8519

Wicks, R. T., Alexander, R. L., Stevens, M., et al. 2016, ApJ, 819, 6

Xiang, L., Wu, D. J., \& Chen, L. 2018, ApJ, 857, 108

Zhao, G. Q., Chu, Y. H., Lin, P. H., et al. 2017b, JGRA, 122, 4879

Zhao, G. Q., Feng, H. Q., Wu, D. J., Chu, Y. H., \& Huang, J. 2017a, ApJL, 847, L8 